\title{
FSM Scenarios of Laminar-Turbulent Transition in Incompressible Fluids
}

\author{
N.M. Evstigneev and N.A. Magnitskii \\ Additional information is available at the end of the chapter
}

http://dx.doi.org/10.5772/48811

\section{Introduction}

The problem of turbulence arose more than hundred years ago to explain the nature of chaotic motion of the nonlinear continuous medium and to find ways for its description; so far it remains one of the most attractive and challenging problems of classical physics. Researchers of this problem have met with exclusive difficulties and there was an understanding of that the problem of turbulence always considered difficult, is actually extremely difficult. This problem is named by Clay Mathematics Institute as one of seven millennium mathematical problems [1] and it is also in the list of 18 most significant mathematical problems of XXI century formulated by S.Smale [2].

The nature of turbulence - the disordered chaotic motion of a nonlinear continuous medium, the causes and mechanisms of chaos generation remain the main issue in the turbulence problem. Several models trying to explain the mechanisms of turbulence generation in nonlinear solid media were suggested at different time. Among such models the most known are Landau-Hopf and Ruelle-Takens models, explaining generation of turbulence by the infinite cascade of Andronov-Hopf bifurcations and, accordingly, by destruction of three-dimensional torus with generation of strange attractor. However, these models have not been justified by experiments with hydrodynamic turbulence.

The universal unified mechanism of transition to dynamical chaos in all nonlinear dissipative systems of differential equations including autonomous and nonautonomous systems of ordinary and partial differential equations and differential equations with delay argument was theoretically and experimentally proven in number of recent papers by the authors [3-12]. The mechanism is developing by FSM (Feigenbaum-Sharkovskii-Magnitskii) scenario through subharmonic and homoclinic bifurcation cascades of stable cycles or stable two dimensional or many-dimensional tori.

In this chapter we are presenting a consistent numerical solution method for 3D evolutionary Navier-Stokes equations with an arbitrary initial-boundary value problem posed. Then we 
consider two well studied problems for incompressible Navier-Stokes equations, namely flow over a backward facing step and Rayleigh-Benard convection in cubic cavity. Numerical solutions of these problems for transitional regimes indicated existence of complicated scenarios formed by theory FSM. Thus, it seems reasonable, that there is no unified laminar-turbulent transition scenario, it can be a cascade of stable limit cycles or stable two dimensional or many dimensional tori, but all these scenarios lay in the frameworks of the FSM-theory.

\section{Construction of high order numerical method for Navier-Stokes equations}

\subsection{Some theorems and assertions}

Here we are talking only about three dimensional evolutionary incompressible Navier-Stokes equations:

$$
\begin{gathered}
\nabla \cdot \mathbf{V}=0, \\
\frac{\partial \mathbf{V}}{\partial t}+(\mathbf{V} \cdot \nabla) \mathbf{V}+\rho_{0}^{-1} \nabla P=v \nabla^{2} \mathbf{V},
\end{gathered}
$$

and suppose that laminar-turbulent transition is well described by these equations. Here $\rho_{0}$ is a constant fluid density, $v$ is the kinematic fluid viscosity, $\mathbf{V}$ is the velocity vector-function and $P$ is the scalar pressure function. Main questions of numerical solution trustworthiness of Navier-Stokes equations and its attraction to real system (1) attractor are:

1. Does the change of infinite dimensional system for finite-dimensional alters the attractor and solution?

2. Do numerical approximation errors have crucial affect on the attractor trajectory?

3. Does a numerical solution convergence to a real solution and how close are they?

Some results of attractor approximation and numerical attractors for nonlinear PDEs were obtained in papers $[13,14]$, but none of them covers such a complicated topic as existence of and numerical trajectories attraction for Navier-Stokes attractors. However in papers [15-19] it is shown that for a well-posed initial-boundary problem there exists an attractor and its dimension and volume is limited from above. This allows us to pose some assumptions on numerical methods that must be used to get a numerical system attractor and that it is close to the attractor of Navier-Stokes equations. To do so we will be following closely to work of R.Temam $[16,20]$.

Existence of a global attractor for 3D Navier-Stokes equations is proven [15, 16]. Let that attractor $A$ exist for a well-posed initial-boundary problem in domain $\Omega \in \mathbb{R}$ with local Lipschitz-contonous boundary $\partial \Omega_{i}$ and velocity vector-function $\mathbf{V} \in \mathbb{R}^{3}$. Let $k_{0}$ be the macroscopic wave number that corresponds with the characteristic length scale $L$ and $k_{k}$ is the Kolmogorov wave number defined as:

$$
k_{k}=\left(\frac{\epsilon}{v^{3}}\right)^{1 / 4}
$$

$v$ - kinematic fluid viscosity and $\epsilon$ - rate of turbulent energy dissipation. whole spectra of wave numbers can be described as: 


$$
k_{i}=\left(k_{0}, k_{1}, k_{2}, \ldots, k_{k}\right), i \in \mathbb{N}
$$

Hence the minimum space dimension that is required to hold all possible flow scales in bounded domain $\mathbf{V} \in \mathbb{R}^{3}$ is defined as:

$$
Y=\left(\frac{k_{k}}{k_{0}}\right)^{3}
$$

The value of $Y$ gives us number of degrees of freedom for the given turbulent flow. We can use the value for $\epsilon$ from [16] where it is shown that $\epsilon$ is limited by the supremum:

$$
\epsilon=v \cdot \lim _{t \rightarrow \infty} \sup \left\{\sup _{\mathbf{v}(x, 0) \subset A} \frac{1}{t} \int_{0}^{t} \sup \left|\nabla^{2} V(x, t)\right|_{x \in \Omega}^{2} d x\right\},
$$

and we can find the following:

Theorem 01. [16] the Hausdorff-Besicovitch dimension of any attractor $A$ in the Navier-Stokes equations for a well-posed boundary value problem is limited by:

$$
\operatorname{dim} A \leq C \cdot \frac{k_{k}}{k_{0}}
$$

where $C$ is an arbitrary constant. One can derive a more explicit estimate. Since:

$$
\epsilon \leq \bar{\epsilon}=v\left|\nabla^{2} \mathbf{V}(x, t)\right|^{2}
$$

and

$$
k_{k} \leq \overline{k_{k}}=\left(\frac{\bar{\epsilon}}{v^{3}}\right)^{1 / 4}=\sqrt{\left(\frac{\left|\nabla^{2} \mathbf{V}(x, t)\right|}{v}\right)}
$$

so taking (4) into account:

$$
\operatorname{dim} A \leq L^{3} \cdot\left(\frac{\left|\nabla^{2} \mathbf{V}(x, t)\right|}{v}\right)^{3 / 2}
$$

where $L$ is a macroscopic problem scale. Since all functions in (9) have finite limits then the dimension of an attractor is always limited from above and its dimension is linked with number of degrees of freedom (4). So we have the following:

Assertion 01. If a well-posed initial-boundary value problem for Navier-Stokes equations in bounded domain $\Omega \in \mathbb{R}^{3}$ with local Lipschitz-continuous boundary $\partial \Omega \in \mathbb{R}^{2}$ has a suitable numerical approximation with degrees of freedom $Y^{*} \geq Y$ that convergence to the given problem on every time step of approximation then the approximated numerical attractor $A^{*}$ converges to the real attractor $A$.

This assertion can be easily verified using estimates for finite-difference and finite-element approximations of Roger Temam [20]. This assertion answers the first question. Now we 
consider the convergence of the numerical method with certain properties for an arbitrary given well posed initial-boundary problem to the real solution of the problem. We are using the following theorem by Roger Temam [20], page 281:

Theorem 02. Let space dimension is 3 and we have some numerical approximation for Navier-Stokes equations with some conditions on which its stable and discrete elements are $h$ for space and $k$ for time. Then there exists the sequence for $h$ and $k \rightarrow 0$, that:

$$
\left.\begin{array}{l}
\mathbf{V}_{h} \rightarrow \mathbf{V} \\
P_{h} \rightarrow P
\end{array}\right\}
$$

strong in $L^{2}(Q)$, week in $L^{\infty}(Q, 0, \Omega) ; Q=\Omega \times[0, t]$.

Here: $\quad \mathbf{V}, P$ - velocity vector-function and pressure scalar-function that correspond to a solution for an initial-boundary value problem, $\mathbf{V}_{h}, P_{h}$ - velocity vector-function and pressure scalar-function on sequence $h$. $C$ is an arbitrary constant. Here we consider one of possible solutions in attractor $A$, since uniqueness of a solution for a problem is not proven in $3 \mathrm{D}$ case. Prove of this theorem is given in [20], p.282, we are using only some conditions for the theorem validity:

Condition 1. If a function $x \mapsto \mathbf{V}(x) ; x \subset \mathbb{R}^{3}$ has the property $\nabla \cdot \mathbf{V}=0$, then a discrete function $h \mapsto \mathbf{V}_{h}$ must maintain the property $\nabla_{h} \cdot \mathbf{V}_{h}=0$.

Condition 2. For any sequence $\mathbf{V}_{h}$ the condition:

$$
\sup \left[\sum_{h}\left|\mathbf{V}_{h+1}-\mathbf{V}_{h}\right|^{k+1}-\sum_{h}\left|\mathbf{V}_{h+1}-\mathbf{V}_{h}\right|^{k}\right] \leq 0,
$$

has to hold.

Appropriate approximations and numerical procedures where used in $[16,20]$. A screw-symmetric numerical operator $\nabla_{h}$ was used for $(\mathbf{V} \cdot \nabla) \mathbf{V}$, linear high order operators where used for linear diffusion parts, etc. Time integration was conducted by implicit Crank-Nicolson method for linear part of Navier-Stokes system and explicit second order for nonlinear part. Pressure correction was used so that Condition 1 is true on each time step. However the theorem is true only for $h$ and $k \rightarrow 0$ [19], i.e. when the numerical system phase space dimension goes to $\infty$. It is obvious that $h$ and $k$ are finite for a real numerical method that is applied on computers. Since the attractor dimension is limited from above (9) and number of degrees of freedom is very large but finite (4), one can stipulate that $h$ and $k$ can be finite if condition (4) and hence (9) are satisfied. It is hard to give a precise estimate but one can approximately evaluate those values by using Kolmogorov turbulent theory for invariant scales.

The least motion scale of turbulence can be defined as $l_{k}=k_{k}^{-1}$, where $k_{k}$ is given by (2). Since $\epsilon$ is bounded (7), the dissipation rate for a developed turbulent flow is given approximately as:

$$
\epsilon \propto \frac{\mathbf{V}^{3}}{L}
$$


One immediately derives:

$$
l_{k}=\left(\frac{v^{3} L}{\mathbf{V}^{3}}\right)^{1 / 4}=\left(\frac{v^{3} L^{4}}{\mathbf{V}^{3} L^{3}}\right)^{1 / 4}=L \cdot R^{-3 / 4},
$$

Hence $Y$ for a developed turbulent regime can be given as:

$$
Y=\left(\frac{L}{L \cdot R^{3 / 4}}\right)^{3}=R^{9 / 4}
$$

that corresponds with the maximum number of degrees of freedom for Direct Numerical Simulation. For smaller values of Reynolds (R) number where the flow is in transitional regime the value for (14) is not perfectly true. For the purpose of finding $Y$ in this case we are using a spatially constructed numerical procedure which is given below. For now we assume that appropriate values of $h$ and $k$ for the given $R$ are defined and conditions 1 and 2 are true for some selected numerical solution procedure. Taking into account that the attractor is a phase space trajectories attraction manifold we have the following

Assertion 02. For a well-posed initial boundary value problem the numerical solution method with correct values of $h$ and $k$ and true Conditions 1 and 2 approximates an attractor of 3D evolutionary Navier-Stokes equations up to the precision order of the numerical method.

\subsection{Numerical method description and consistent mesh adaptation}

So we can construct the numerical method with the above mentioned properties and it can be used to analyze laminar-turbulent transition as a nonlinear dynamic system. However we should point out that bifurcation parameters do depend on the numerical method. So the values of these parameters can vary from one method to another for a given bifurcation. We are considering advection initial boundary problems with no external force acting for dimensionless Navier-Stokes equations. The general problem can be described as: Let $\Omega$ be a bounded domain with local Lipschiz-continuous boundaries $\partial \Omega_{i}$. One must find velocity vector-function $\mathbf{V}: \Omega \times[0, t] \rightarrow \mathbb{R}^{3}$ and scalar pressure function $P: \Omega \times[0, t] \rightarrow \mathbb{R}$ such as:

$$
\begin{gathered}
S h \cdot \frac{\partial \mathbf{V}}{\partial t}+(\mathbf{V} \cdot \nabla) \mathbf{V}+E u \cdot \nabla P=\frac{1}{R} \nabla^{2} \mathbf{V} \text { in } Q=\Omega \times(0, t) ; \\
\nabla \cdot \mathbf{V}=0 \text { in } Q ; \\
\mathbf{V}=f(\vec{x}), \text { on } \partial \Omega_{0} \times(0, t), \mathbf{V}=0, \text { on } \partial \Omega_{1} \times(0, t), \frac{\partial \mathbf{V}}{\partial \vec{n}}=0, \text { on } \partial \Omega_{2} \times(0, t) ; \\
\mathbf{V}(\vec{x}, 0)=\mathbf{V}_{0}(x, y, z) \text {, in } \Omega \text { with } \nabla \cdot \mathbf{V}_{0}(x, y, z)=0 .
\end{gathered}
$$

Similarity criteria are constructed using characteristic macroscopic scales: $S h=f L / V$ Strouhal number; $E u=2 P /\left(\rho V^{2}\right)$ - Euler number; $R=V L / v$ - Reynolds number; $L$ - macroscopic characteristic scale, $f$ - frequency, $V$ - characteristic velocity, $P_{0}$ - reference pressure, $\rho=$ const - fluid density, $v$ - fluid kinematic viscosity. Various boundary conditions are given as: $\partial \Omega_{0}$ - inflow condition, $\partial \Omega_{1}$ - solid wall condition, $\partial \Omega_{2}$ - outflow condition and the problem is initialized with initial conditions. Most important similarity criterion is Reynolds number for laminar-turbulent transition. The rest criteria can be used to scale some real problems using $\pi$-theorem [21] and are not used since we are interested only in nonlinear 
dynamics of equations with no regard to a real specific problem. In order to determine necessary number of discrete elements one should take into account two factors; the first is to use the upper bound (14) as a start and derive $\epsilon$ from the averaged equations and the second is to use modified wave number analysis to calculate how many elements are needed to represent certain wave number and thus necessary number of degrease of freedom. Using this method, see [22] one can numerically determine anisotropic element density in $\Omega$ and optimize this number. This approach also satisfies all conditions of theorems and assertions described above. Since the rate of energy dissipation is a function of Reynolds number and particular initial-boundary conditions we are going to compute required $Y$ using Reynolds averaging. Let us rewrite momentum equation in (15) in coordinates as:

$$
\frac{\partial V_{i}}{\partial t}+\sum_{j}\left(V_{j} \frac{\partial V_{i}}{\partial x_{j}}\right)+\frac{1}{\rho} \frac{\partial P}{\partial x_{i}}=v \frac{\partial^{2} V_{i}}{\partial x_{i}^{2}} ; i, j=\{1,2,3\}
$$

and introduce averaging:

$$
\mathbf{V}=\overline{\mathbf{V}}+\mathbf{V}^{\prime}
$$

thus:

$$
\overline{V_{i}+V_{j}}=\overline{V_{i}}+\overline{V_{j}} ; \overline{\partial_{\alpha} V}=\partial_{\alpha} \bar{V} ; \overline{\overline{V_{i}} V_{j}}=\overline{V_{i} V_{j}} ; \alpha=\{t ; x\}
$$

Here $\bar{V}$ - averaged and $V^{\prime}$ instantaneous functions of velocity vector-function. By applying (17) to (16) one gets:

$$
\frac{\partial \overline{V_{i}}}{\partial t}+\sum_{j}\left(\overline{V_{j}} \frac{\partial \overline{V_{i}}}{\partial x_{j}}+\overline{V_{j}^{\prime} \frac{\partial V_{i}^{\prime}}{\partial x_{j}}}\right)+\frac{1}{\rho} \frac{\partial \bar{P}}{\partial x_{i}}=\sum_{j} \frac{\partial \overline{\tau_{i j}}}{\partial x_{j}}
$$

where second rank tensor $\tau_{i j}=v\left(\frac{\partial V_{i}}{\partial x_{j}}+\frac{\partial V_{j}}{\partial x_{i}}\right)$ corresponds to the Newtonian fluid. Multiplying (16) on $\overline{V_{i}}$ and applying (17) one gets:

$$
\overline{\frac{\partial V_{i}}{\partial t} V_{i}}+\sum_{j}\left(\overline{V_{j} \frac{\partial V_{i}}{\partial x_{j}} V_{i}}\right)+\frac{1}{\rho} \overline{\frac{\partial P}{\partial x_{i}} V_{i}}=\sum_{j} \overline{\frac{\partial \tau_{i j}}{\partial x_{j}} V_{i}}
$$

Multiplying (19) on $\overline{V_{i}}$ :

$$
\frac{\overline{\partial V_{i}}}{\partial t} \bar{V}_{i}+\sum_{j}\left(\overline{V_{i} V_{j}} \frac{\partial \overline{V_{i}}}{\partial x_{j}}\right)+\frac{1}{\rho} \frac{\partial \bar{P}}{\partial x_{i}} \overline{V_{i}}=\sum_{j}\left[\left(\frac{\partial \overline{\tau_{i j}}}{\partial x_{j}}+\frac{\partial T_{i j}}{\partial x_{j}}\right) \overline{V_{i}}\right],
$$

one gets the stress equation, where $T_{i j}=-\overline{V_{i}^{\prime} V_{j}^{\prime}}$ are the components of virtual (Reynolds) stress tensor. Subtraction of (21) from (20) reads:

$$
\overline{\frac{\partial V_{i}^{\prime}}{\partial t} V_{i}^{\prime}}+\sum_{j}\left(\overline{V_{j} \frac{\partial V_{i}}{\partial x_{j}}} \overline{V_{i}}-\overline{V_{j}} \frac{\partial \overline{V_{i}}}{\partial x_{j}} \overline{V_{i}}\right)+\frac{1}{\rho} \frac{\partial \overline{P^{\prime}}}{\partial x_{i}} \overline{V_{i}^{\prime}}=\sum_{j}\left(\frac{\partial \overline{\tau_{i j}}}{\partial x_{j}} \overline{V_{i}^{\prime}}-\frac{\partial T_{i j}}{\partial x_{j}} \overline{V_{i}}\right),
$$


where summation is performed in accordance with (18). The value $\overline{\tau_{i j}{ }^{\prime} V_{i}^{\prime}}$ is the second infinitesimal order to the other parts of (22) and then the latter can be simplified by using averaging rules (18) as:

$$
\begin{array}{r}
\frac{1}{2}\left(\overline{\left.\frac{\left(V_{i}^{\prime}\right)^{2}}{\partial t}+\sum_{j} \frac{\partial \overline{\left(V_{i}^{\prime}\right)^{2}}}{\partial x_{j}} \overline{V_{j}}\right)+\frac{1}{\rho} \frac{\overline{\partial P^{\prime}}}{\partial x} V_{i}^{\prime}}+\frac{1}{2} \sum_{j} \frac{\partial \overline{V_{i}^{\prime 2} V_{j}^{\prime}}}{\partial x_{j}}+\right. \\
+\sum_{j}\left(\overline{\frac{\partial V_{i}^{\prime}}{\partial x_{j}} \tau_{i j}^{\prime}}+\overline{V_{j}^{\prime} V_{i}^{\prime}} \frac{\partial \overline{V_{i}^{\prime}}}{\partial x_{j}}\right)=0 .
\end{array}
$$

One can get the equation for perturbation energy balance applying a summation of (23) by index $i$ :

$$
\frac{\partial k}{\partial t}+\sum_{j} \frac{\partial k}{\partial x_{j}} \overline{V_{j}}=-\sum_{j}\left(\frac{\partial}{\partial x_{j}}\left(\rho^{-1} \overline{P^{\prime} V_{j}^{\prime}}+\sum_{i}\left[\overline{V_{i}^{\prime 2} V_{j}^{\prime}}\right]\right)\right)+\sum_{i, j}\left(\overline{T_{i j}} \frac{\partial \overline{V_{i}^{\prime}}}{\partial x_{j}}\right)-\epsilon,
$$

where perturbation kinetic energy is written as:

$$
k=\frac{1}{2} \sum_{i} \overline{\left(V_{i}^{\prime}\right)^{2}}
$$

and perturbation rate of dissipation is described by:

$$
\epsilon=\sum_{i, j}\left(\overline{\frac{\partial V_{i}^{\prime}}{\partial x_{j}} \tau_{i j}^{\prime}}\right)=\frac{v}{2} \sum_{i, j} \overline{\left(\frac{\partial V_{i}^{\prime}}{\partial x_{j}}+\frac{\partial V_{j}^{\prime}}{\partial x_{i}}\right)^{2}} .
$$

The latter expression (26) is the exact value of (7) for a given Reynolds number. We are using the following correlation to define the Kolmogorov wave number (2):

$$
k_{k}=\left(\frac{R^{2}}{2} \sum_{i, j} \overline{\left(\frac{\partial V_{i}^{\prime}}{\partial x_{j}}+\frac{\partial V_{j}^{\prime}}{\partial x_{i}}\right)^{2}}\right)^{1 / 4},
$$

where the sum expression is numerically calculated by a test simulation with the maximum possible number of discrete elements, defined by (14). After that we are constructing the isolines of $N Y$, calculated by (4), where the dissipation wave number is applied through (27) and $N$ is defined by modified wave number analysis, described bellow. Then the mesh that we are using is adopted to satisfy all calculated values of $Y$. Only after all these procedures we can say that dynamic nonlinear analysis results we obtained are true and can be considered trustworthy.

In order to solve an initial-value problem for Navier-stokes numerically we are introducing the following semi discrete scheme based on the fractional step method using high order TVD 
Runge-Kutta forth order method with each step of RK as:

$$
\begin{aligned}
& 1 . \mathbf{V}^{\prime}-\mathbf{V}^{n}=-\Delta t\left(\mathbf{V}^{n} \cdot \nabla\right) \mathbf{V}^{n} ; \\
& \text { 2. } \mathbf{V}^{\prime \prime}-\mathbf{V}^{\prime}=\Delta t \Theta \nabla^{2} \mathbf{V}^{\prime(\prime)} \\
& \text { while } \nabla \cdot \mathbf{V}^{\beta+1} \neq 0 ;\left\{\begin{array}{l}
3 . \nabla^{2} P=-\nabla \cdot \mathbf{V}^{\beta} / \Delta t \\
4 . \mathbf{V}^{\beta+1}=\mathbf{V}^{\prime \prime}-\Delta t \nabla P
\end{array}\right. \\
& \text { 5. } \mathbf{V}^{n+1}=\mathbf{V}^{\beta+1}
\end{aligned}
$$

Here $\mathbf{V}^{n}$ and $\mathbf{V}^{n+1}$ are the previous timestep and next timestep values of the velocity vector function; $P$ is the pressure, $\Delta t$ - timestep for the given Runge-Kutta stage and $\Theta$ is the diffusion parameter and for a forced advection problems equals $R^{-1}$. All other superscripts on $\mathbf{V}$ are intermediate values of velocity vector function inside the stage. More details on this numerical procedure can be found in $[9,23,24]$. Each step here is just shortly described. On step 1 in (28) advection equations are solved with the condition (11) satisfied. We are using fifth order WENO-type scheme. Several problems were solved for pure advection equation and Burgers equation (in 1D and 2D) before the final variant of WENO scheme was selected. Since the timestep is limited by the accuracy requirements the numerical scheme was explicit. On the second step of (28) diffusion equations are solved by using large stencil approximation with the 6-th order of accuracy. It is possible to apply implicit method here (in (28) in brackets) for natural advection problem described bellow, since values of $\Theta$ are of unity magnitude. But for forced advection where $\Theta=R^{-1}$ (for $R>100$ ) it is possible to apply explicit time method. Academician Belotserkovsky O.M. [25] suggested a physical interpretation of this fractional step method. Step 1 and 2 are calculating not solenoidal vector field that breaks the mass conservation equation. But if we apply the operator $(\nabla \times)$ on step 1 and 2 for both (28) and Navier-Stokes equations (15), we get the curl transport equations, since $\nabla \times \nabla P=0$. Curl properties are correctly simulated though $\nabla \cdot \mathbf{V}=0$ is not satisfied even for steps 1 and 2 . The latter corrected by applying the pressure correction on the third step, where the Poisson equation is solved for pressure scalar function until the solenoidal criteria is met up to the machine accuracy. After that the velocity field is corrected and he velocity vector function is solenoidal on step 4 up to machine accuracy, so condition 1 for correct approximation is satisfied.

Spatial discretization is a combined finite volume for $\mathbf{V}$ and finite element for $\mathrm{P}$ discretization. Since we are focusing on fundamental problems (with simple geometry) the discrete elements are rectangular cuboids, thus it allows us adjusting its dimensions in accordance with the calculated values of $Y$. Finite elements are the same cuboids but variables are stored on vertexes rather than centers of mass as for $\mathbf{V}$. So the described system of equations is rewritten for arbitrary convex element $i$ with volume $W_{i}$ and $\Omega=\bigcup_{i=1}^{N} W_{i} ; W_{i} \cap W_{j}=$ for $\forall i \neq j$ as:

$$
\begin{gathered}
\iiint_{W_{i}} \nabla \cdot \mathbf{V} d W=\frac{1}{W_{i}} \oint_{S_{i}} \mathbf{V}^{f} \cdot \vec{n} d S=0, \\
\frac{\partial}{\partial t} \iiint_{W_{i}} \mathbf{V} d W+\oint_{S_{i}}\left[\mathbf{V}^{f} \mathbf{V}^{f} \cdot \vec{n}\right] d S+\oint_{S_{i}} / P^{f} / \vec{n} d S-\Theta \oint_{S_{i}}[\nabla \mathbf{V}]^{f} d s=0,
\end{gathered}
$$

here $S$ - is one of element side square, subscript $f$ refers to the face value and $\vec{n}$ is the unit vector on the side, total number of sides is B. Using discrete elements and applying summation 
instead of integration

$$
\oint_{S_{i}} f(U) \cdot \vec{n} d S \approx \sum_{j=1}^{B} f\left(U_{j}\right) \cdot \vec{n} \Delta S_{j}
$$

one immediately reads:

$$
\begin{gathered}
\sum_{j=1}^{B}\left[\mathbf{V}_{j}^{f} \cdot \vec{n}_{j}\right] \Delta S_{j}=0, \\
W_{i} \frac{d}{d t} \mathbf{V}_{i}+\sum_{j=1}^{B}\left[\mathbf{V}_{j}^{f} \mathbf{V}_{i}^{f} \vec{n}_{j}\right] \Delta S_{j}+/ \nabla_{h} P /-\Theta \sum_{j=1}^{B}\left[\nabla_{h} \mathbf{V}\right]_{j}^{f} \cdot \vec{n} \Delta S_{j}=0 .
\end{gathered}
$$

Here / / operator is treated by finite element method. Then discrete system (30) is applied in (28) by described numerical methods. Please note that $(\mathbf{V} \cdot \nabla) \mathbf{V}=\nabla \cdot(\mathbf{V V})+\mathbf{V} \nabla \cdot \mathbf{V}=$ $\nabla \cdot(\mathbf{V V})$ in (29) and (30) since $\nabla \cdot \mathbf{V}=0$ on every timestep.

In order to complete the analysis of numerical scheme and answer all questions positively one must perform modified wave number analysis for used discrete schemes. We are considering first order PDE like on step 1, in (28). Let the differential operator $\partial_{x}$ be approximated by central differences as $\partial_{x}=\delta_{x}+\mathcal{O}\left(\Delta x^{2}\right)$ using $N$ discrete segments each with the length $\Delta x$ and let the function be $u(x)=c_{k} \cdot \exp (i k x)$. One immediately gets analytical solution as $\partial_{x} u(x)=i k c_{k} \exp (i k x)=i k u(x)$. For the given central differences approximation the real part $u(x)=\cos (k x)$ becomes:

$$
\begin{aligned}
\delta_{x}(\cos (k x))=\frac{1}{2 \Delta x} \cos (k(x+\Delta x)) & -\frac{1}{2 \Delta x} \cos (k(x-\Delta x))= \\
& =-\left(\frac{\sin (k x)}{\Delta x}\right) \sin (k x)
\end{aligned}
$$

Here we can see that the difference of analytical and numerical solutions is in wave number $k$ that changes to $k^{\prime}=\sin (k x) / \Delta x$ and if $k \Delta x<<1$ then $k^{\prime}=k-k^{3} \Delta x^{2}+\ldots$. For small $k$ the result of approximate solution (31) is close to the analytical. But when wave number $k$ increases i.e. discretization scale $\lambda$ decreases $(\lambda=2 \pi / k$ and if $\Delta x=\lambda / N$ then $k \Delta x=2 \pi / N)$, the error grows. So we call $k^{\prime}$ a modified wave number by the numerical scheme. Thus by applying this analysis one can get the minimal undisturbed scale representation of the given numerical discrete scheme. This allows us getting minimal number of finite volumes necessary for the given $k$ say calculated $k_{k}$. In general for the given complex function one can write:

$$
k^{\prime} \Delta x=-i \sum_{j=0}^{N-1} c_{j} \exp \left(\frac{i 2 \pi j k}{N}\right)=-i \sum_{m=-s}^{m=s} a_{m} \exp (i m k \Delta x),
$$

where $s$ is half length of the stencil in discrete space and $a_{m}$ - are the interpolation coefficients of the considered numerical scheme. One can immediately see from (32) that for symmetric schemes only real part presents in the modified wave number since $a_{m}=-a_{-m}$ and $a_{0}=0$. That explains why symmetric approximation cannot be used for approximation of advection operator in (30). Numerical wave number analysis for WENO scheme was first made in [26] ], but here we use analytical analysis. By applying WENO weights, since WENO scheme is fifth order everywhere (even for discontinuous functions, see [24, 27] one can get interpolation 
coefficients:

$$
a_{m}^{W E N O}=(-1 / 30 ; 1 / 4 ; 11 / 5 ;-1 / 2 ;-1 / 20 ; 0) ; s=3 ; \mathcal{O}\left(\Delta x^{5}\right) .
$$

By inserting (33) into (32) one can get modified wave number (real and imaginary parts) and derive minimal necessary numbers of elements to represent a given wave number, see fig.1. So one can see that for the 2 -nd order central differences scheme at least 8 elements are needed
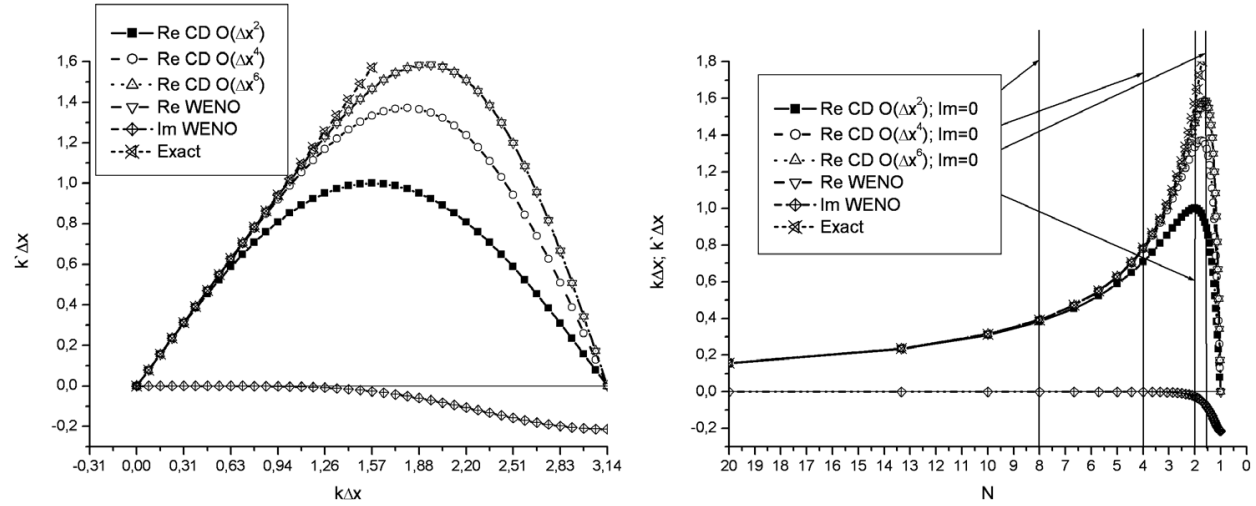

Figure 1. Modified wave number for different schemes to $k \Delta x \in[0, \pi]$ (left) and minimal number of elements $N$ for the correct given wave number representation (right). CD - central differences.

to describe a given wave number correctly, and 2 elements for the 6-th order scheme as well as for the WENO scheme. So now we know that the values that are found in accordance with (27) must be multiplied by two. Applying the same method for other parts of discretization one can point out that the given estimate for two elements is enough and here is omitted for the sake of brevity.

Time integration also requires some care since the condition (11) is in spatio-temporal condition and if CFD literature is known as TVD (TVB for equals sign) condition for nonlinear PDEs $[28,29]$. Since WENO approximation is TVB, we are going to give a TVD condition (11) for time integration:

$$
\frac{\partial V_{i}}{\partial t}=L\left(V_{i}\right)
$$

where $L$ is the given complex nonlinear TVB differential operator consisting of all steps (28). Simple TVD method for (34) is the Euler's method $V^{n+1}=V^{n}+\Delta t L\left(V^{n+s}\right)$. If (34) is the explicit method (i.e. $s=0$ ) then the stability criterion is a Courant number $\Delta t \leq C$. One can give the following

Lemma 01. If a direct Euler's method (34) is applied for a TVB or TVD spatial approximation operator for $\Delta t \leq C$ and by applying $m$-stage Runge-Kutta scheme

$$
\begin{gathered}
V^{n+1}=\sum_{i=1}^{m}\left(\alpha_{i} V^{n+1-i}+\Delta t \beta_{i} L\left(V^{n+1-i}\right)\right) ; \\
\sum_{i=1}^{m} \alpha_{i}=1, \forall \alpha_{i}, \beta_{i} \geq 0, \text { on } L(V),
\end{gathered}
$$

then the solution is stable in any norm 
$\left\|V^{n+1}\right\| \leq \max \left\{\left\|V^{n}\right\| ;\left\|V^{n-1}\right\| ; . . ;\left\|V^{n-m+1}\right\|\right\}$

with time step limited by:

$$
\Delta t \leq \min _{i} \frac{\alpha_{i}}{\beta_{i}} \Delta t(\text { Euler })
$$

if $\forall \beta_{i}=0$, then $\frac{\alpha_{i}}{\beta_{i}} \rightarrow \infty$.

The same idea is given in [30]. Proving the lemma is done by considering the combinations of Euler's steps. This lemma gives us constants $\alpha_{i}$ and $\beta_{i}$ that were used to construct the forth order TVD Runge-Kutta method that fulfills the condition (11) and has a maximum stable timestep possible. One should point out that the standard RK4 method with constants $(1 / 6 ; 1 / 3 ; 1 / 3 ; 1 / 6)$ is not TVD and can't be used for time integration. So it is shown that the presented numerical method is guaranteed to satisfy all given theorems, conditions and assertions and, hence, can be used to describe nonlinear dynamics of laminar-turbulent transition.

The whole work described here took three and half years and $80 \%$ of the time was used on calculation. New CUDA technology [31] was applied lately and now all described numerical methods are calculated using NVIDIA GPUs that greatly accelerate the research.

\section{Laminar-turbulent transition for the flow over a backward facing step}

One of the best studied problems is the problem of the flow over a backward facing step. It was simulated by many different authors and has lots of benchmark results and even few results about nonlinear dynamics in the problem.

\subsection{Initial-boundary value problem, mesh adaptation and benchmark verification}

The geometry and boundary conditions are taken from [32] with small adaptation, since laminar-turbulent transition is investigated as a dynamic system so we compare not only benchmark results but bifurcation sequences as well.

Domain $\Omega$ with local Lipschiz-continuous boundaries $\partial \Omega_{i}$ is represented by a rectangular channel divided by two unequal parts in Cartesian coordinates. The length of the domain spans in $\mathrm{X}$ axis direction. The first part of the channel has length $L_{1}=2.0$, the second part of the channel length is $L_{2}=10.0$, so the whole length of the domain is $L=L_{1}+L_{2}=12.0$. Height spans in $\mathrm{Y}$ axis and the second part of the domain is higher than the first one by the size of the step $h=0.6$ with the first part height of $H=0.9$. So the second part of the domain height is $H+h=1.5$. Width of the domain $W=3.5$ spans in $Z$ axis direction and is the same for both part. The geometry is almost identical to [32] with $L_{1}=10.0$. The formed step causes the flow to create recirculation zones inside $\Omega$ and transit to turbulence with the growth of Reynolds number.

There are many different boundary conditions available in papers for this particular problem [33-35, 38, 39]. All boundaries in step $\mathrm{Y}$ axis direction $\partial \Omega_{1} \in \min _{y}(\Omega)$ are given by solid walls with no slip condition. The upper boundary $\partial \Omega_{2} \in \max _{y}(\Omega)$ is given as either wall or symmetry. Boundaries $\partial \Omega_{3} \in \max _{z}(\Omega)$ and $\partial \Omega_{4} \in \min _{z}(\Omega)$ vary from one work to another. The boundary $\partial \Omega_{3}$ is given as a solid wall but $\partial \Omega_{4}$ as symmetry plane in [33]. Boundaries in 
$\mathrm{Z}$ direction in some other papers, i.e. [35, 40], are given by symmetry planes thus modeling a semi-2D problem and decreasing number of mesh elements due to the lack of side boundary layers. We choose boundary conditions like in [32] where boundaries in $\mathrm{Z}$ direction are no slip walls. Boundary $\partial \Omega_{5} \in \min _{x}(\Omega)$ is the inflow boundary where Poiseuille laminar solution for $\mathrm{R}=200$ in the channel with the same width and height. Boundary $\partial \Omega_{6} \in \max _{x}(\Omega)$ is the outflow boundary. We introduce smooth outflow boundary conditions [41] with the outflow buffer so that unphysical outflow conditions are neglected. Pressure is set on the outflow boundary as a reference value. Initial conditions in whole domain $\Omega$ for velocity vector-function and scalar pressure function obtained by laminar stationary solution of this problem with $R=200$ so that $\nabla \cdot \mathbf{V}=0$ at $\mathbf{t}=0$. Reynolds number is defined as:

$$
R=V^{0} L / v, L=2 W H /(W+H) .
$$

Here $V^{0}$ is the inflow velocity normalized by the flow rate, like in [32]. Due to the fact that DNS is very computational demanding we are limiting the maximum $\mathrm{R}$ by analyzing literature results. It is shown in [32] that a "chaotic attractor" that corresponds to noise frequencies band appear at $R>1500$ so we set maximum $R=1500$. For the purpose of minimizing computational efforts we are considering three meshes adopted for $R=700, R=1000$ and $R=1500$. The rest Reynolds number meshes are lying inside the segment. Using an upper bound estimate (14) and taking modified wave number analysis in account one gets total mesh numbers as: 5040 813( $R=700), 11246$ 827( $R=1000)$ and $24004984(R=1500)$. We set $L=1 \frac{3}{7}$ from the given geometry and since we know $R$, we can evaluate minimal Kolmogorov linear scale of one element $l_{k}$ :

$$
l_{k}=\{0.024495281 ; 0.018745885 ; 0.013830488\},
$$

and, hence, in Cartesian coordinates we have $M=\{491 ; 641 ; 869\}, N=\{62 ; 81 ; 109\}, K=$ $\{144 ; 188 ; 254\}$, elements for the upper bound estimate. Time step is selected 0.005 for all calculations [? ]. We performed these three control calculations on fine grids and calculated isosurfaces of minimal scales $l_{k}$ by (27). Example of these isosurfaces for $R=1000$ is shown in fig.2.

It is clearly visible that we must use fines mesh with element size of 0.01577 of boundary layers and recirculation zone, but the rest of the flow has s much lighter demand for small scales. So final meshes where chosen to be:

$$
\begin{aligned}
& G 1=\{245 X 54 X 83\}, R=700 ; \Delta x_{\alpha}=\{0.0677 ; 0.01957 ; 0.03964\} \approx 103^{3} ; \\
& G 2=\{339 X 72 X 119\}, R=1000 ; \Delta x_{\alpha}=\{0.04696 ; 0.01577 ; 0.028548\} \approx 143^{3} ; \\
& G 3=\{560 X 100 X 177\}, R=1500 ; \Delta x_{\alpha}=\{0.04696 ; 0.01577 ; 0.028548\} \approx 215^{3} ;
\end{aligned}
$$

where cubic values are shown for required memory estimate. After that all other calculations for the given problem are performed on these (39) three grids that satisfy necessary conditions of theorems. In order to perform benchmark tests boundaries $\partial \Omega_{3,4}$ where changed accordingly. Values of are the same $l_{k}$ for inside flow region and near periodic boundaries so our grid resolution (39) is overdensed for test calculations with periodic boundaries. So new mesh was generated in the same manner through calculation of $l_{k}$ but it contained less number of $l_{k}$ elements, so benchmark calculations took less time to solve. 


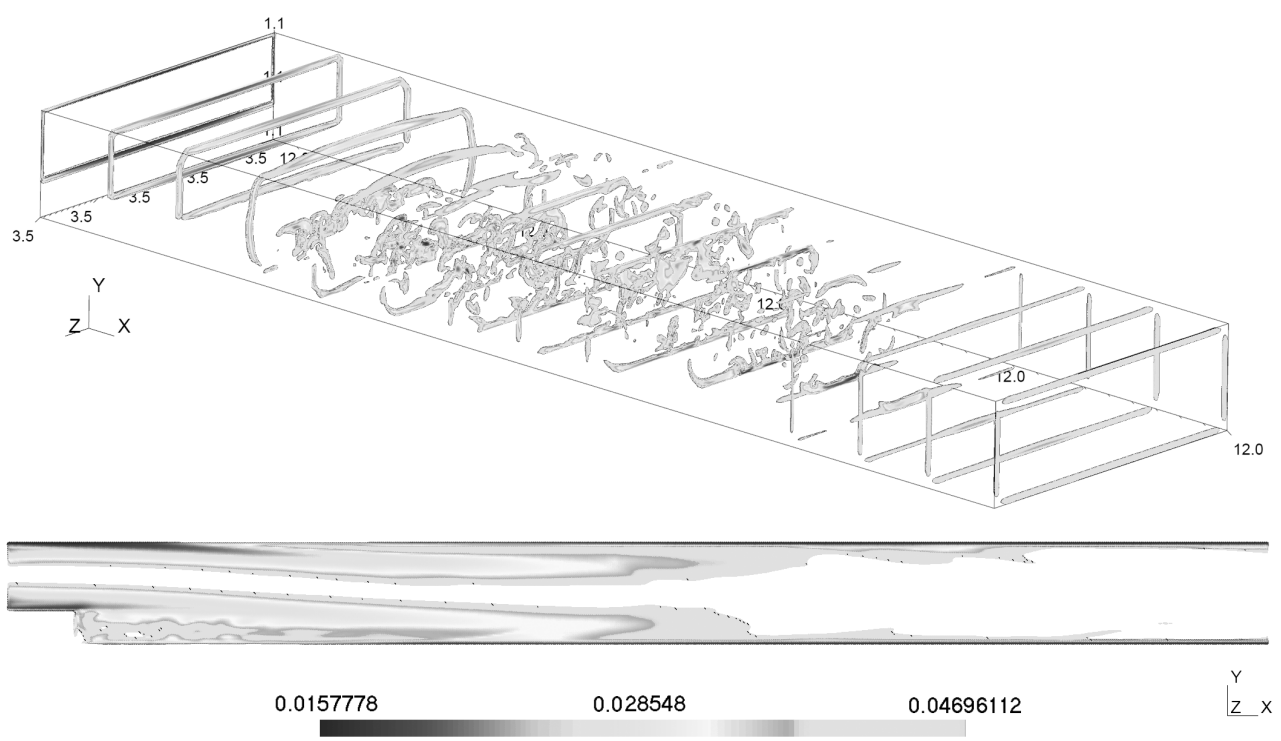

Figure 2. Calculated values of $l_{k}$ for $R=1000$. Central section and literal 3D sections are shown.

While solving various modifications of the problem we changed height of the step in accordance with the geometry presented for particular benchmark tests and here all detail data of these geometries is omitted due to brevity. Integral and statistical data of $\mathbf{V}$ and $P$ functions is considered as well as friction wall coefficients.

First we overseen qualitative comparison of results for $\mathrm{R}=1500$ with side walls with DNS [35], [42], [37] and physical model [43] results. Velocity isolines sections are presented in fig.3. One can clearly see the 3D structure of the flow plus the recirculation vertexes that appear near all walls. This agrees well with the presented papers. To compare qualitatively periodic boundary results we used papers [44] and [35]. The flow is much more simple for periodic boundaries in $\mathrm{Z}$ direction.

For the quantitative analysis we considered some papers: [44] for $R=\{100 ; 389 ; 1000\}$, [34] for $R=150-800$, [35] for $R=1500$, [45] in $C_{f}$ comparison for $R=5500$ (using LES model) and $[38,46]$ for virtual stress tensor comparison for $R=1800$ (using LES model). All data agreed well for benchmarks with maximum difference of $7 \%$. Data was extracted from papers with high precision using shareware GetData Graph Digitizer 2.24.

Detail results are omitted for the sake of brevity, but some results are presented here. Reattachment vortex length ration to the height of the step is presented in fig.4 and compared with results from various sources, i.e. [34]. One can clearly see that the results are well agreed with reference data.

More quantitative results are presented in fig. 5, where a mean wall friction coefficient is presented. The dynamic eddy viscosity model was used to solve the flow for $R=5500$, and was compared with other LES and DNS results. One can see that the result agree well with presented data, especially with DNS data (rectangles) from [45]. 


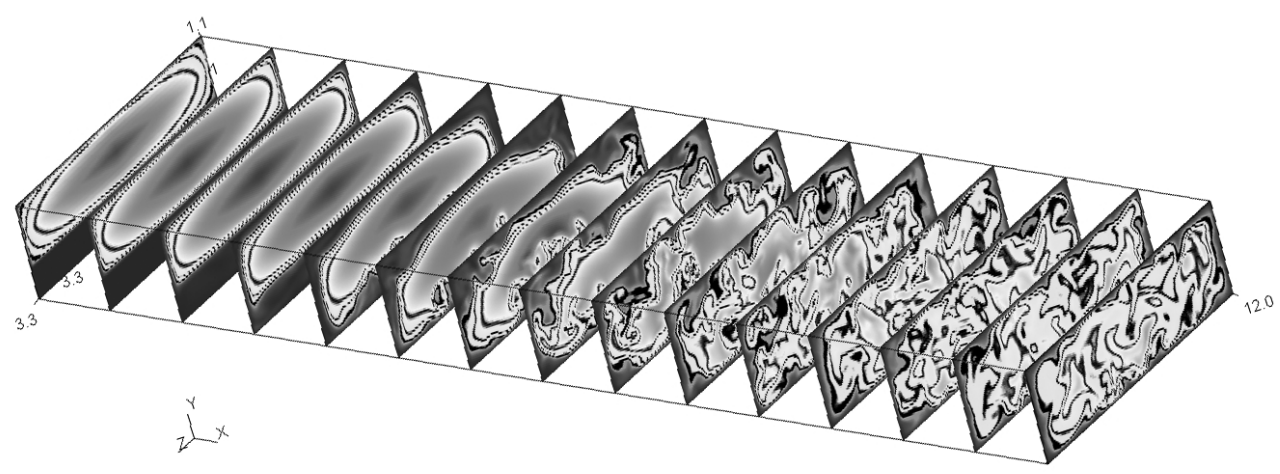

Figure 3. Instantaneous velocity vector-function modulus isolines in sections for $\mathrm{R}=1500$.

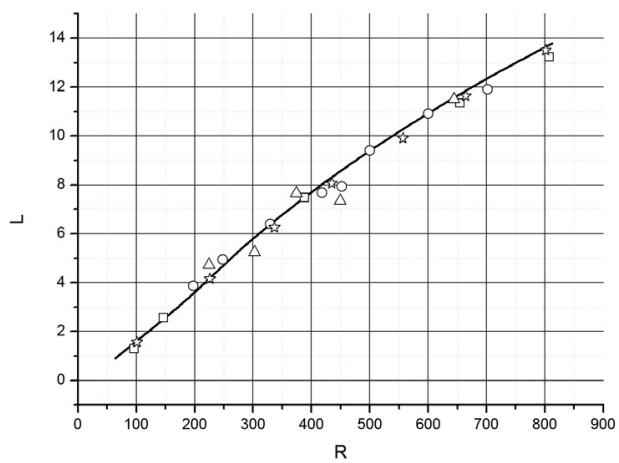

Figure 4. Mean reattachment length to the step height, compared with reference data, line - current method

Comparison for Reynolds stress correlations at $R=1800$ with data from [38] presented in fig.6. One can see a very good agreement between DNS and experimental data.

More benchmark details are omitted for the sake of brevity, see [23, 47].

\subsection{Nonlinear dynamics of laminar-turbulent transition}

The idea of analysis was used many times in our research $[7,8,23,48]$ and corresponds with [32]. We saved data for velocity function in format $V_{x}, V_{y}, V_{z}$. Saving data for all domain was impossible since there's not enough disk space (it requires about 6,43 terabytes for one fixed Reynolds number), so we choose several points and saved data from them. Points in Cartesian coordinates normalized by length in each direction are: $p 1=\{0.1,0.5,0.5\}$; $p 2=\{0.2,0.5,0.5\} ; p 3=\{0.5,0.5,0.5\} ; p 4=\{0.7,0.5,0.5\} ; p 5=\{0.8,0.1,0.1\}$. We are using Reynolds number as the bifurcation parameter for the problem and forming sets of three dimensional phase subspaces of the whole infinite dimensional phase space. The subspaces 


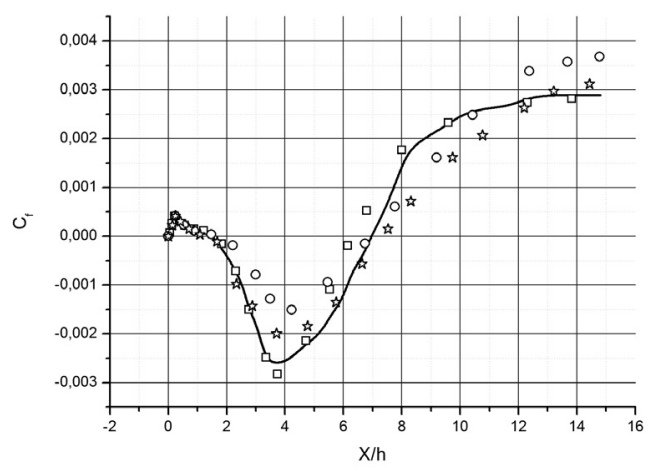

Figure 5. Mean wall friction coefficient $C_{f}$ for $R=5500$, line - current method, pints - reference data
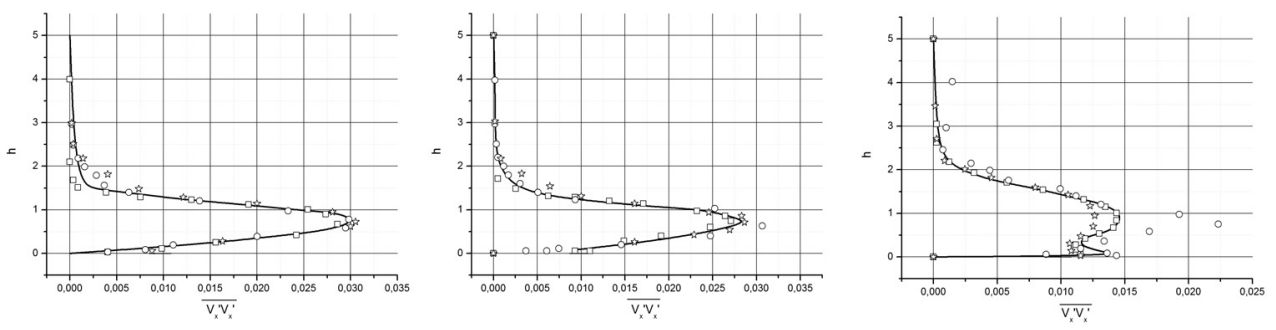

Figure 6. Reynolds stress correlations comparison for $R=1800$ with experimental data at length $x / h=\{4 ; 6 ; 15\}$ in the center $(\mathrm{W} / 2)$.

are formed by velocity vector components. Under infinite dimensional phase space here we consider finite dimensional phase space produced by the numerical system (30) whose space dimension is greater than the attractor dimension of the system (15) for the given initial-boundary value problem with a finite preset Reynolds number. It is important to notice that there exists a hysteresis of a solution if one approaches a fixed $\mathrm{R}$ from different sides, say solution $G$ exists for $R_{0}$ if we travel to it by $R_{1} \rightarrow R_{0}, R_{1}<R_{0}$ but does not exist for $R_{0}$ if $R_{1}>R_{0}$, i.e. see [49]. So we are only considering the following cascade of bifurcation parameters $R_{1}<R_{2}<\ldots<R_{n}=1500$.

The solution is laminar for $R$ from 100 up to 736 but the time of stationary solution formulation increases as $R$ grows. For the stationary laminar solution we can monitor a fixed point in infinite dimensional phase space and in all five three dimensional subspaces of velocity vector function. Starting from a system exhibits one frequency mode regime. A limited cycle $C_{1}$ is formed from every stationary point in phase space and has projections in all subspaces. One can see a projection of the cycle for the point $P_{1}$ in fig. 7 .

At the point of $R=850$, the cycle looses stability and forms a stable two dimensional invariant torus $T_{2}=C_{1} \otimes C_{2}$ as the result of Andronov-Hopf bifurcation. This torus is located in all infinite dimensional phase space and can be found in all subspaces for points $P_{i}$. Presumably, this is due to the fact of incompressibility what expresses in elliptic operator for pressure 


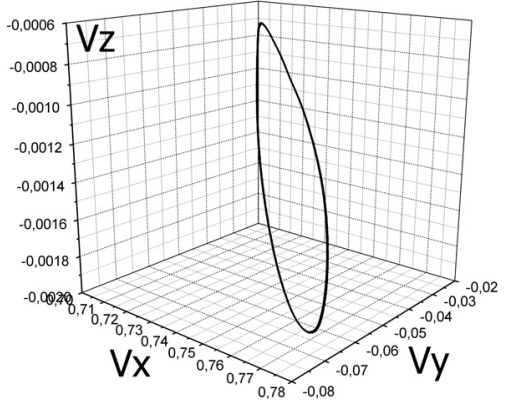

Figure 7. Point $P_{1}$, stable limit cycle, $\mathrm{R}=740$

and hence the acoustic speed is infinite. An example of this torus is shown in fig.8. It is clearly seen that the system is very sensible for the values of bifurcation parameters. It is shown in fig.8, that a change of $R$ by 1.0 changes the attractor from $C_{1}$ to $T_{2}$. Comparing these results with the mentioned work [32] we can say that the formation of the first cycle appears almost at the same Reynolds number, here we have $R=737$ and in [32] the value is $\mathrm{R}=735$. The formation of two-frequent mode in [32] appears at $\mathrm{R}=855$, but there's no stable phase space trajectory available, since numerical errors started dominating. And further investigation in [32] is performed using frequency analysis that indicated creation of the other independent frequency. The recent report in TsAGI [50] of Sibgatullin I.N. indicated that other initial-boundary problems exhibit the resembling scenario by which an invariant torus is formatted in phase space. But all reports and papers indicate that a chaotic behavior follows the formation of two dimensional invariant tori. We suppose that this is due to the fact that numerical methods, used in the papers and reports, don't meet necessary conditions for theorems and assertions outlined here and so we continued the numerical analysis.
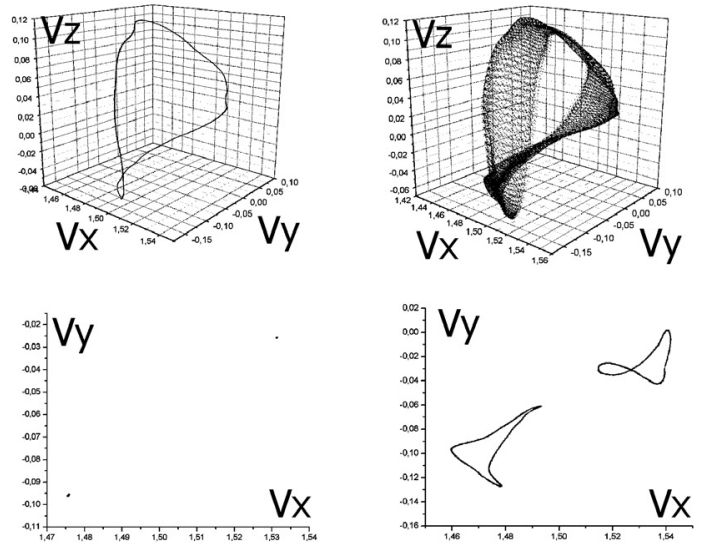

Figure 8. $R=849$ Phase subspace cycle projection and section, point P4(left); $R=850$ Phase subspace torus projection and section, point $\mathrm{P} 4$ (right) 
Further increase of $R$ leads to more complex topology of cycles that are forming the torus. The process of this complication is shown in fig. 9 at point $P_{3}$ and in fig. 10 at point $P_{5}$.
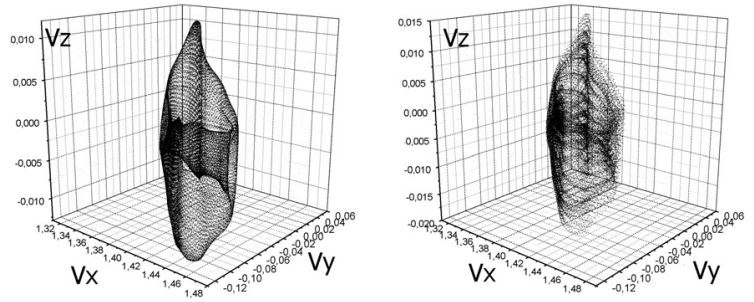

Figure 9. Torus complication in subspace projection at point $P_{3} . R=\{851 ; 883\}$
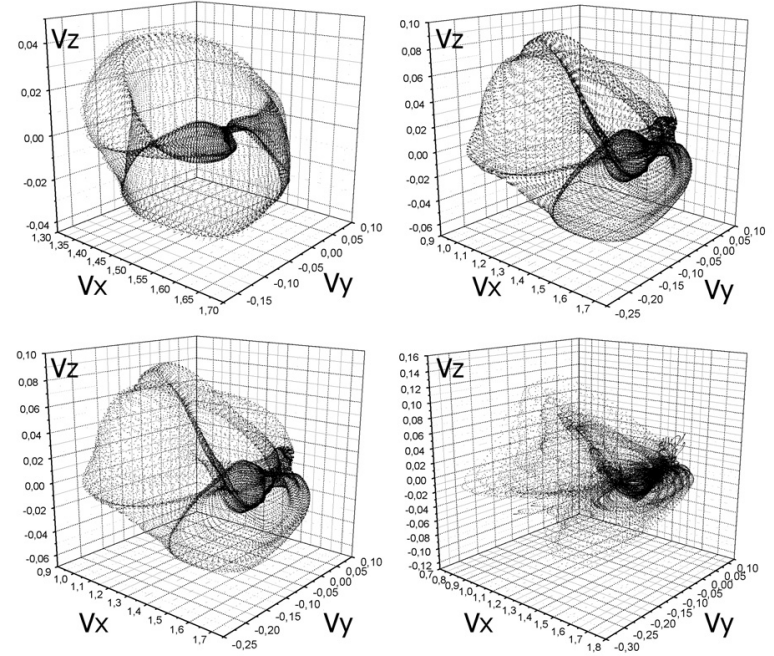

Figure 10. Torus complication in subspace projection at point $P_{5}, R=\{851 ; 882 ; 883 ; 885\}$

This process continues up to $R=883$. It was found that at this point the two dimensional torus looses stability in the whole infinite dimensional space and forms a three dimensional invariant torus through the Andronov-Hopf bifurcation. This torus is formed by the topological multiplication of three irrational frequency cycles $T_{3}=C_{1} \otimes C_{2} \otimes C_{3}$. Its projections in different subspaces are shown in fig.9, 10 for $R \geq 883$.

Since the space dimension in one point of the phase subspace is the same as the formed torus dimension one should increase the phase subspace dimension. It can be clearly seen on sections fig.11 where a new "coiling" can be monitored around the old cycle in plane section.

To do so we are taking data from another point thus forming a four dimensional subspace and then we are performing two sections by two dimensional planes. The first section in $P_{5}$ (plus data from $P_{2}$ marked with asterisk) is shown in fig.12 on the left. The torus structure can be seen by performing another section by the plane $W=0.005$. One can see the three dimensional torus structure in fig. 12 in sections for $\mathrm{R}=883$. 

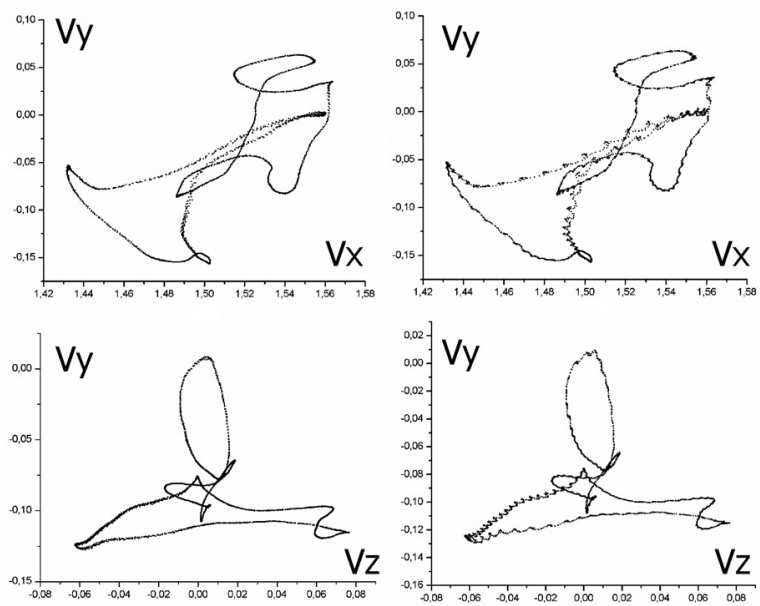

Figure 11. Plane sections of phase subspaces in $P_{4}$ and $P_{5}$ for $R=(882.5 \rightarrow 883)$ from left to right
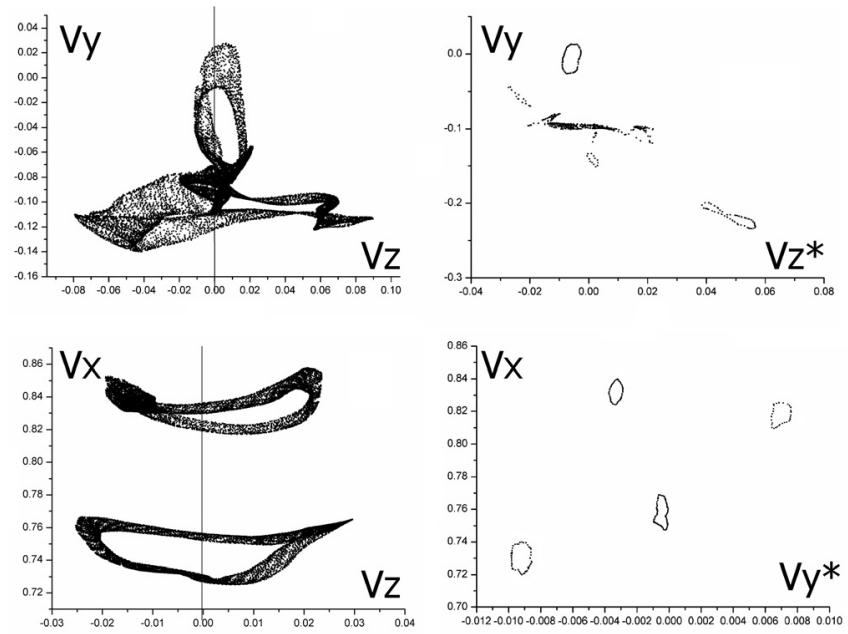

Figure 12. Section of the phase subspace in Point $P_{3}$ (top) and $P_{5}$ (bottom) plus $P_{2}$ data (marked asterisk), $R=883$, on the left. Section of the four dimensional phase subspace by the additional plane, on the right

Three dimensional invariant torus losses its stability and through the doubling period bifurcation forms a double period three dimensional torus for $R=883.8$ It requires huge amount of data to get results shown in fig.13. Depicted results required about $375 \mathrm{~Gb}$ of data per one point and could only be completed by numerical methods on GPUs or on massive parallel clusters. All different sections are presented in fig.13 for four dimensional phase subspaces at $P_{3}+P_{2}$ and $P_{5}+P_{2}$.

It can be clearly seen that the torus topology is more complicated, i.e. see fig.13 at point $P_{5}$, and fig. 12 - second section, although the bifurcation parameter only changed by $0.091 \%$. These 

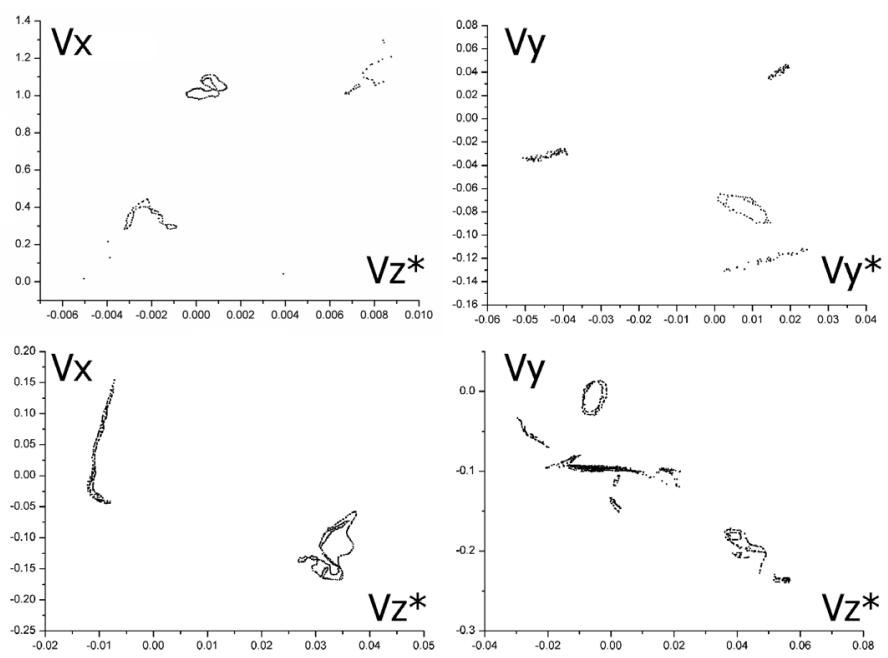

Figure 13. Section of the four dimensional phase subspaces by additional planes at point $P_{3}+P_{2}(*)$ (top) and $P_{5}+P_{2}(*)$ (bottom)

results show that a double period three dimensional torus is formed in all infinite dimensional phase space for $R=883.8$. Further investigation is impossible due to the exponential growth of bifurcations. The second section view is already next to incomprehensible for $R=890$ and consists of a filled black squire. In conclusion we found the following scenario of laminar-turbulent transition:

$$
C_{1} \rightarrow T_{2} \rightarrow T_{3} \rightarrow T_{3 \otimes 2} \rightarrow,
$$

It can be seen in (40) that the scenario exhibits initial stage of Landau-Hopf scenario (Hopf bifurcation cascades) and initial stage of FSM scenario, since the Feigenbaum scenario is progressing after the three dimensional torus. It corresponds with the initial stage of FSM scenario. One should point out that a three dimensional torus is stable at least for the time of numerical simulation for $1,5 \cdot 10^{7}$ timesteps at $R=883$.

\section{Laminar-turbulent transition for Rayleigh-Benard convection}

One of the best studied and widely analyzed problems of fluid mechanics is the Rayleigh-Benard natural convection problem. The problem has been considered by many and has lots of results in the field of nonlinear bifurcation analysis, analytical, numerical and experimental.

\subsection{The Oberbeck-Boussinesq approximation of Rayleigh-Benard convection, dimensionless form and benchmarks}

One of the possible mathematical models for this problem is the Oberbeck-Boussinesq approximation. Here we are closely following [51] and assume, that fluid physical properties $(\nu, \beta)$ are only linear functions of temperature perturbations. The fluid density can be given 
as a function of temperature perturbation as:

$$
\rho=\rho_{0}\left(1-\beta\left(T-T_{0}\right)\right),
$$

where $\rho$ - fluid density, $T$ - fluid temperature, $\beta$ - fluid thermal expansion coefficient, $\rho_{0}, T_{0}$ - mean values of fluid density and temperature. It is assumed under Oberbeck-Boussinesq approximation that density only changes due to temperature difference and, thus, causing buoyancy, yet fluid is considered incompressible. Temperature emission due to friction is also neglected. Introducing (41) to Navier-Stokes equations (1), assuming temperature passive advection-diffusion and taking gravity vector in Cartesian coordinates as $\vec{g}=\{0 ; 0 ;-1\}$ in account, one gets:

$$
\begin{aligned}
\nabla \cdot \mathbf{V} & =0, \\
\frac{\partial \mathbf{V}}{\partial t}+(\mathbf{V} \cdot \nabla) \mathbf{V}+\rho_{0}^{-1} \nabla P & =v \nabla^{2} \mathbf{V}+\vec{g} \beta\left(T-T_{0}\right), \\
\frac{\partial T}{\partial t}+\mathbf{V} \nabla T & =\chi \nabla^{2} T,
\end{aligned}
$$

here $\chi$ is a fluid thermal conductivity coefficient. There are many scales can be chosen that make (42) dimensionless for the Rayleigh-Benard convection problem. One of the most common ways [51] is to use time scale $\tau$ as $\tau=h^{2} / v$, where $h$ is the length between two planes with given temperature difference. Another way is to associate $\tau$ with the momentum transport by viscous terms and then $\tau=h^{2} / v$. Introducing additional dimensionless similarity criteria one can formulate a Rayleigh-Benard convection problem: find vector-function $\mathbf{V}: \Omega \times[0, t] \rightarrow \mathbb{R}^{3}$, scalar pressure function $P: \Omega \times[0, t] \rightarrow \mathbb{R}$ and and scalar temperature function $T: \Omega \times[0, t] \rightarrow \mathbb{R}$ that satisfy the following initial-boundary value problem:

$$
\begin{gathered}
\frac{\partial \mathbf{V}}{\partial t}+(\mathbf{V} \cdot \nabla) \mathbf{V}+\nabla P=\nabla^{2} \mathbf{V}+\operatorname{RaPr}^{-1}\left(T-T_{0}\right) \cdot(0 ; 0 ;-1)^{T} \text { in } Q ; \\
\nabla \cdot \mathbf{V}=0 \text { in } Q=\Omega \times(0, t) ; \\
\frac{\partial T}{\partial t}+\mathbf{V} \nabla T=\operatorname{Pr}^{-1} \nabla^{2} T \text { in } Q ; \\
\mathbf{V}=0, \partial T / \partial \vec{n}=0, \text { on } \partial \Omega_{0} \times(0, t) ; \mathbf{V}=0, T=T_{\alpha} \text { on } \partial \Omega_{1} ; \\
\mathbf{V}(\vec{x}, 0)=\mathbf{V}_{0}(\vec{x}), \nabla \cdot \mathbf{V}_{0}=0 ; T(\vec{x}, 0)=T_{0}(\vec{x}) \text { in } \Omega .
\end{gathered}
$$

Here: $\Omega$ is a bounded domain with local Lipschiz-continuous boundary $\partial \Omega_{i} ; t$ is time; $T$ is a fluid temperature; $T_{0}$ is a reference fluid temperature; $\operatorname{Pr}=v / \chi$ is the dimensionless Prandtl number; $R a=g \beta h^{3} \Delta T /(v \chi)$ is the Rayleigh number; $h$ is height in $\Omega$ between $\partial \Omega_{1} ; \Delta T$ is a temperature difference between $\partial \Omega_{1}$.

There are two main types of boundary conditions. First consider boundary conditions whose plane is parallel to the temperature gradient, i.e. $\Omega_{0}$. One usually chooses either periodic boundary conditions for temperature and velocity or wall boundary conditions with Neumann type for temperature. On other planes, namely $\Omega_{1}$, temperature gradient by Dirichlet boundary conditions is set with wall no slip boundary for velocity.

Numerical solution method differs from (28) only in temperature equation and in diffusion part of Navier-Stokes equations. We applied implicit five diagonal matrix solution method [52] for all diffusion parts of (43) thus accuracy drops from 6-th order to 4-th order in space. It can be shown by the modified wave number analysis that number of elements remains the same just with a little lost of accuracy. In order to solve matrix equation $[A][X]=[B]$ that arises from implicit method for diffusion parts of equation a five diagonal fast factorization 
solution routine is adopted for CPU calculations [53] and Geometric Multigrid method for GPU [54].

In order to satisfy conditions of the theorems we must adopt mesh by introducing Reynolds number through Prandtl and Payleigh numbers. We are using paper results $[55,56]$ that indicate the following relation is true:

$$
R \simeq R a^{0.44} \operatorname{Pr}^{-0.76},
$$

for the range of $0.9<\operatorname{Pr}<2$ and $1 \cdot 10^{5}<R a<1 \cdot 10^{9}$. It is also known [57, 58] that transition form "soft" turbulence (where some frequencies can be determined in frequency analysis) to "hard" turbulence (where frequency-amplitude response becomes a coloured noise) occurs at $R a_{c r} \approx 4 \cdot 10^{7}$ for $\mathrm{Pr}>0.9$. Assuming that it is impossible to make any quantitative analysis after similarity criteria greater than critical values we take relation (44) and find Reynolds number as:

$$
R_{\text {max }} \simeq R a_{c r}^{0.44} \min \left(P r_{c r}\right)^{-0.76}=2856 .
$$

Using (45) and applying it to (27) one can get the following mesh adopted variants for cubic and cylindrical domains after calibration simulations:

$$
\begin{aligned}
& G 1=\{250 \times 250 X 250\} \text { for } \operatorname{Pr}=0.9 ; \Delta x_{\max }=6.1 \Delta x_{\text {wall }} ; \\
& G 2=\{216 \times 216 \times 216\} \text { for } \operatorname{Pr}>1.0 ; \Delta x_{\max }=5.2 \Delta x_{\text {wall }} ; \\
& G 3=\{185 \times 185 X 185\} \text { for } \operatorname{Pr}>1.25 ; \Delta x_{\max }=5.0 \Delta x_{\text {wall }} ; \\
& G 4=\{250 X 250 X 100\} \text { for } \operatorname{Pr}>4.0 ; \Delta x_{\max }=4.5 \Delta x_{\text {wall }} ; r / h=4 ; \\
& G 5=\{400 X 400 X 47\} \text { for } \operatorname{Pr}>5.0 ; \Delta x_{\max }=3.5 \Delta x_{\text {wall }} ; / h=14 ;
\end{aligned}
$$

where $r$ is the cylinder radius and $h$ is the cylinder height. Immersed boundary is used for cylindrical approximation, for more information see [59]. For other geometry domains grid is specified in the same manner and omitted here for the sake of brevity.

Benchmarking the problem requires many different domain configurations and different dimensionless variations of the equations (43). We skip that for the sake of brevity and can recommend book by professor Getling A.V. [51] for more information on dimensionless forms, physical background and analytical analysis. It is known from the linear minimodal approximation of the problem [51] that the flow with wall boundary conditions on $\Omega_{0}$ is more stable than that with periodic conditions or two dimensional problems. For the infinite $\mathrm{Pr}$ number the critical value of Rayleigh criterion for the first instability is $R a_{c r}=1707.762$ for the wave number $k=3.117$ in case of wall boundary conditions and $R a_{c r}=27 \pi^{4} / 4 \approx 657.511$ with $k=2.221$ for periodic boundary conditions. For benchmark verification several papers where considered [60].

Solution in cylindric domain for $R a=2000-31000$ and $r / h=4$ with zero initial conditions is shown in fig.14, top and with initial perturbation $V_{z}=\cos (2 \pi z /(2 r)) / 100-$ in fig.14 bottom, where one can see the appearance of absolutely different solution for perturbed initial conditions with the prototype function $\cos (k x)$, see [51].

Some results in rectangular domain where also considered and compared with [61] with very good agreement. More results where compared with [51,60] for $r / h=14$ for range of 

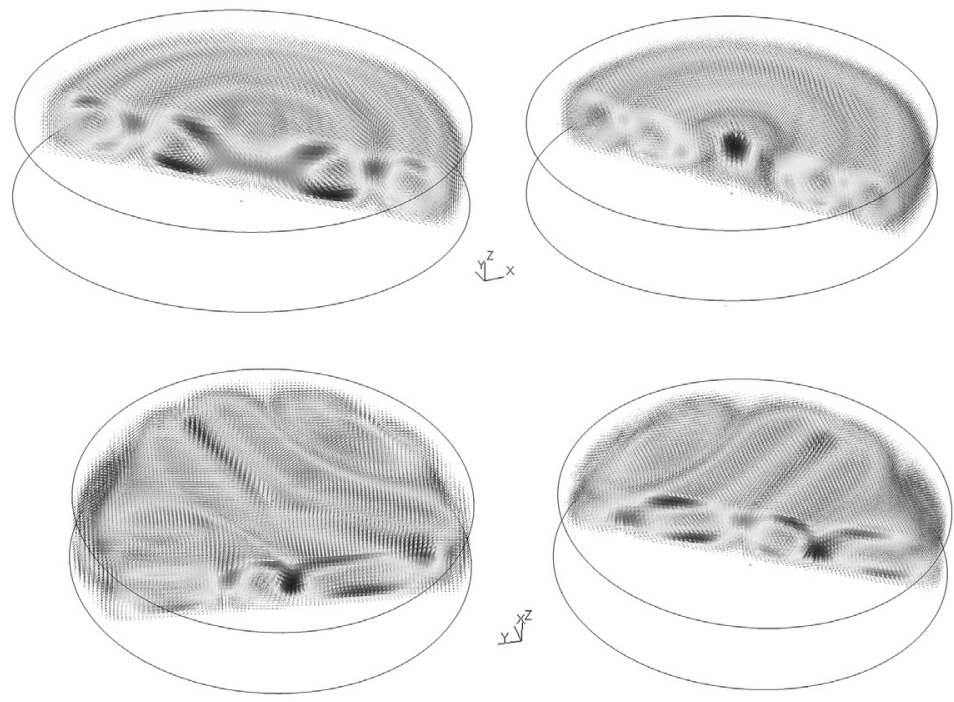

Figure 14. Velocity vector function section $R a=2000-31000, r / h=4$. Zero initial conditions on the top and perturbed initial conditions on the bottom.

$R a=\{2565,4500,6500,16850\}$. We could clearly see the process of different pattern formation (round, circular, "vetruvian man", hexagon, irregular). For the analysis of nonlinear dynamics we considered a cubic domain absolutely identical to the physical experiment, presented in the book by P.G. Frik [62]. Experiment was made in a copper cubic domain with the height of $40 \mathrm{~mm}$. Horizontal boundaries where thermally stabilized and vertical boundaries formed a constant gradient. The experiment was aimed to investigate frequencies and attractors using thermal differential pares. Numerical simulation for $R a=2 \cdot 10^{5}$ and $\operatorname{Pr}=7$ is shown in fig.15.
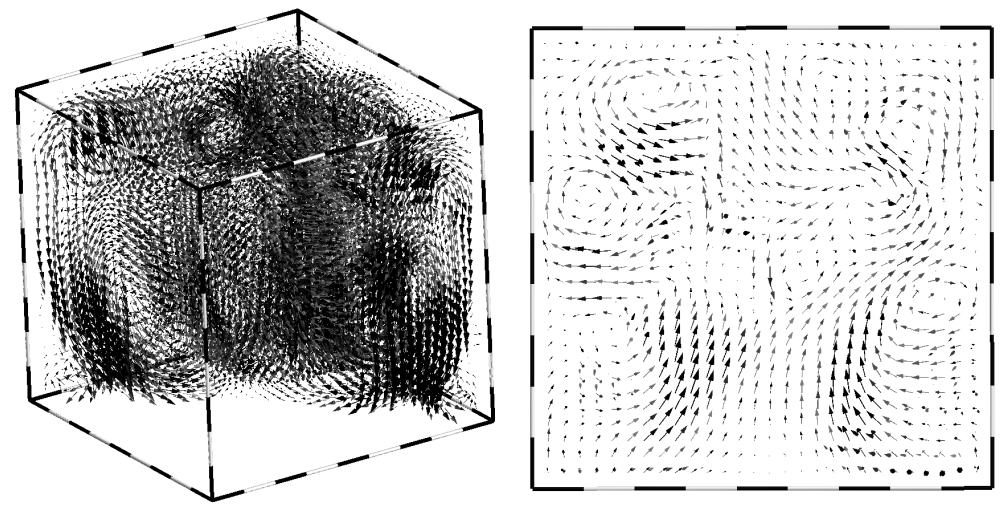

Figure 15. Velocity vectors in $3 \mathrm{D}$ and plane section $(1 ; 0 ; 0)$, for $R a=2 \cdot 10^{5}$ and $\operatorname{Pr}=7$. Every six vector is shown. 


\subsection{Nonlinear dynamics of laminar-turbulent transition.}

The analysis is absolutely identical to the one used for the problem of the flow over backward facing step. For this point we select five points with relative Cartesian coordinates $p_{1}=\{0.5 ; 0.5 ; 0.5\} ; p_{2}=\{0.4 ; 0.5 ; 0.5\} ; p_{3}=\{0.5 ; 0.8 ; 0.5\} ; p_{4}=\{0.8 ; 0.5 ; 0.667\} ; p_{5}=$ $\{0.16 ; 0.167 ; 0.1\}$. The subspaces of infinite dimensional phase space are constructed by velocity vector functions in various points and their combination. Similarity criteria of $R a$ and $\operatorname{Pr}$ numbers are used as bifurcation parameters. We took fixed values for Prandtl number and increased $R a$ number for every $P r$ number, thus we considered five various series of calculations.

\subsubsection{First calculation series.}

Prandtl number is set as 1.866666666 . While we change $R a$ from 1 to 14100 we could see the formation of various stationary solutions with various recirculation zones formation in the domain with the complete correspondence to [62]. Laminar solution can be seen for $R a<$ $2.5 \cdot 10^{5}$ in the whole domain, that corresponded to the fixed stationary point in the infinite dimensional phase space and in all subspaces. However this fixed point jumped from one position to another in the phase space as a function of $R a$ number. This stationary point looses stability for $R a=2.5 \cdot 10^{5}$ and a limited cycle is formed in the phase space and in each phase subspace. This cycle projection for point $p_{1}$ is shown in fig 16.
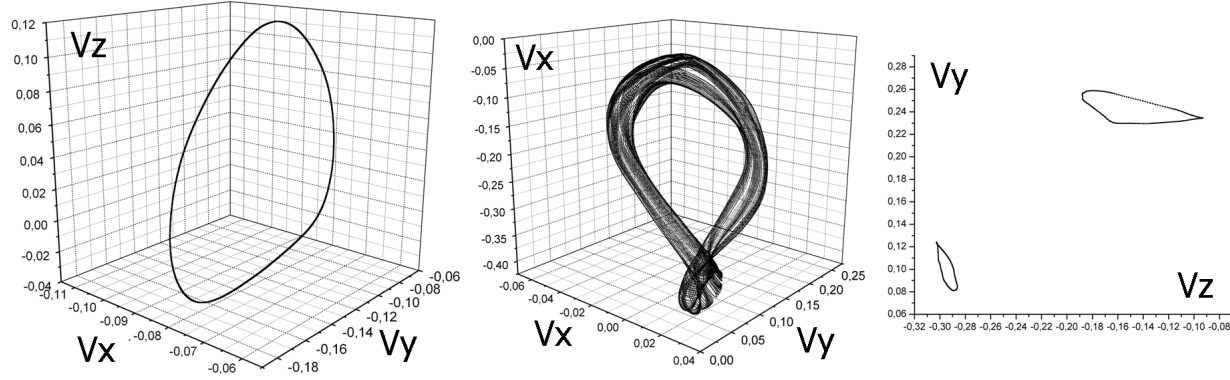

Figure 16. Phase 3D subspace and its sections for $\operatorname{Pr}=1.86666$. From left to right: limited cycle, point $p_{1}, R a=2.5 \cdot 10^{5}$; two dimensional invariant torus, point $p_{1}$; Poincare section of the torus, $R a=2.67820 \cdot 10^{5}$.

This cycle looses stability at $R a=2.67820 \cdot 10^{5}$ and forms two dimensional invariant torus through the Andronov-Hopf bifurcation. This torus projection in the three dimensional phase subspace and its plane section are depicted in fig. 16. This torus is lying in the whole infinite dimensional phase space and has projections in all of its subspaces.

The two-dimensional tori of double and quadruple periods are formed with the further increasing of Rayleigh number (fig. 17 and fig.18 ). Point $p_{5}$ in fig.18 depicts the torus projection near wall boundaries for $R a=2.9133225210 \cdot 10^{5}$.

Further increasing of Ra number led to chaotic instability. This can be related to the limited accuracy of the numerical method and solution trajectories slipped from one to another in such sensible dynamic system. The cascade of bifurcations agreed well with experimental 

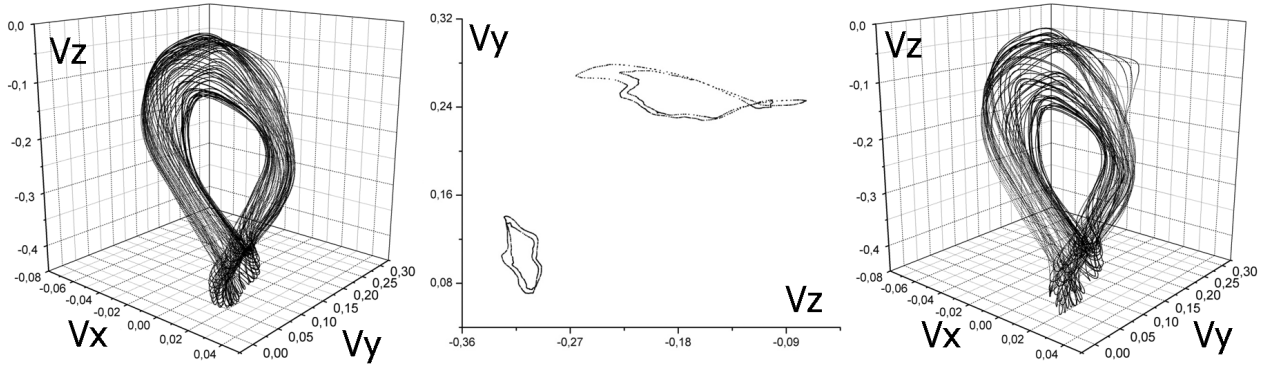

Figure 17. Phase 3D subspace and its sections for $\mathrm{Pr}=1.86666$. From left to right: double period two-dimensional invariant torus, point $p_{1}, R a=2.767858170 \cdot 10^{5}$; Poincare section of the double period torus; quadruple period two-dimensional invariant torus, point $p_{1}, R a=2.9133225210 \cdot 10^{5}$.
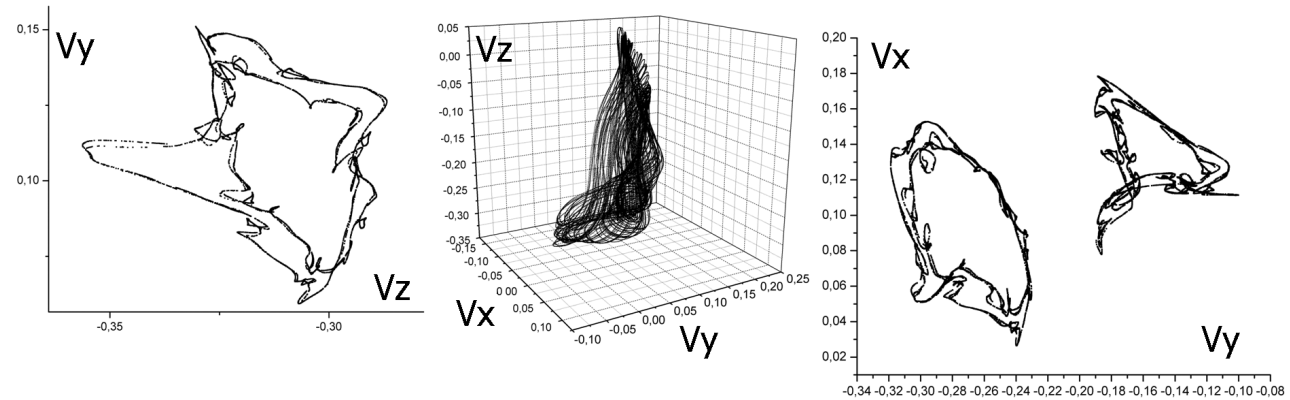

Figure 18. Phase 3D subspace and its sections for $\operatorname{Pr}=1.86666$. From left to right: $1 / 2$ (zoomed) Poincare section of the quadruple period two-dimensional torus, point $p_{1}, R a=2.9133225210 \cdot 10^{5}$; quadruple period two-dimensional invariant torus, point $p_{5}, R a=2.9133225210 \cdot 10^{5}$; Poincare section of the quadruple period torus, point $p_{1}$.

results from [62] with only difference in exact values for $R a$ number. In [62] it could be clearly seen the formation of limit cycle and torus (two irrational frequencies), but further investigations led to chaos so it was impossible to tell from the experimental data of further bifurcations for the formed torus.

\subsubsection{Second calculation series.}

$\operatorname{Pr}$ number is fixed as 1.61290 . The fluid motion is stationary up to $R a=1.361 \cdot 10^{6}$ when a limit cycle is formed from the stationary point in the whole infinite dimension phase space. Further increasing of $R a$ up to $1.365 \cdot 10^{6}$ led to the doubling period bifurcation with formation of double period limit cycle and, immediately, formation of the two-dimensional invariant torus through the Andronov-Hopf bifurcation.

This torus can be clearly seen for $R a=1.366 \cdot 10^{6}$ on the 3D subspace projection to the $\left(V_{x}, V_{y}\right)$ plane in fig.19. Another doubling period bifurcation occurs at $R a=1.36905 \cdot 10^{6}$ forming a 

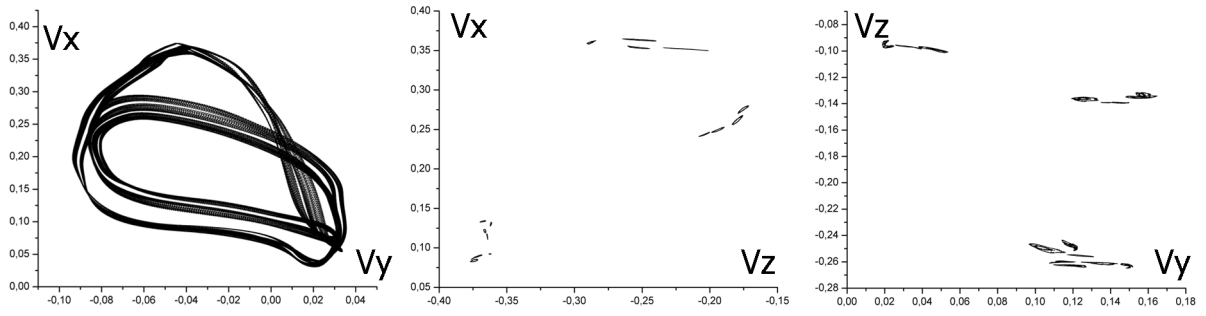

Figure 19. Phase projection and its sections for $\mathrm{Pr}=1.6129$. From left to right: the two dimensional invariant torus projection on $\left(V_{x}, V_{y}\right)$ plane for point $p_{1}, R a=1.366 \cdot 10^{6}$; Poincare section of the torus at point $p_{1}$; Poincare section of the double period torus at point $p_{1}, R a=1.36905 \cdot 10^{6}$.

double period two-dimensional invariant torus that can be seen in fig.19. Further analysis was limited due to the numerical noise.

\subsubsection{Third calculation series.}

Prundtl number is set 1.354839. The limit cycle in system phase space is formed from the stationary point at $R a=1.286 \cdot 10^{6}$ and its projection on $\left(V_{x}, V_{y}\right)$ plane is shown in fig.20.
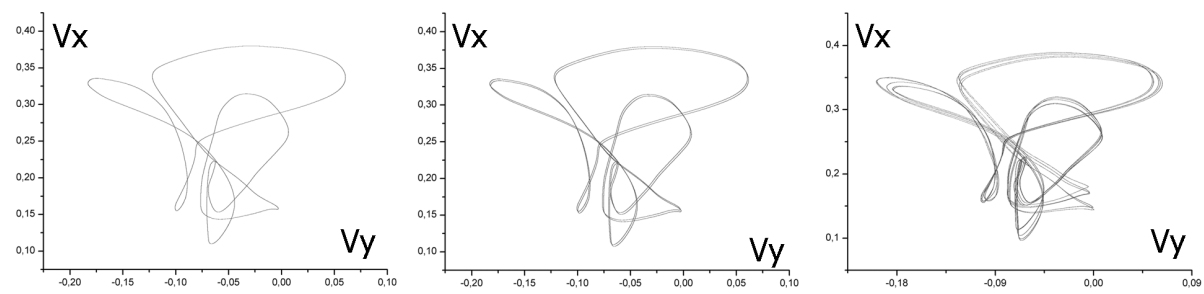

Figure 20. Phase subspace projection to $\left(V_{x}, V_{y}\right)$ plane for $\operatorname{Pr}=1.354839$ at point $p_{1}$. From left to right: stable cycle, $R a=1.286 \cdot 10^{6}$; double period stable cycle, $R a=1.296 \cdot 10^{6}$; quintuple period stable cycle, $R a=1.306 \cdot 10^{6}$.

Next doubling period bifurcation occurs at $R a=1.296 \cdot 10^{6}$ with the formation of double period limit cycle, see fig.20. This cycle is lying in all infinite dimensional phase space and in all subspaces. With the increasing of $R a$ number the cycle suffers cascades of bifurcations in accordance with the FSM scenario. The quintuple period cycle is formed for $R a=1.306 \cdot 10^{6}$ who's projection on $\left(V_{x}, V_{y}\right)$ plane is shown in fig.20.

A triple period cycle can be seen at $R a=1.308 \cdot 10^{6}$ in all subspaces and its projection on $\left(V_{x}, V_{y}\right)$ plane is shown in fig.21. It means that the system suffered the whole subharmonic cascade of bifurcations and now there are all other unstable cycles exist in the system in accordance with the theory FSM. In conclusion one can say that there exist multiple scenarios of laminar-turbulent transition in Rayleigh-Benard convection as functions of $\mathrm{Pr} / \mathrm{Ra}$ ratio:

1. Landau-Hopf bifurcation scenario that forms sets of many-dimensional tori $T^{n}$.

2. Landau-Hopf bifurcation scenario with doubling period bifurcation on n-dimensional invariant torus (Landau-Hopf scenario + FSM scenario). 

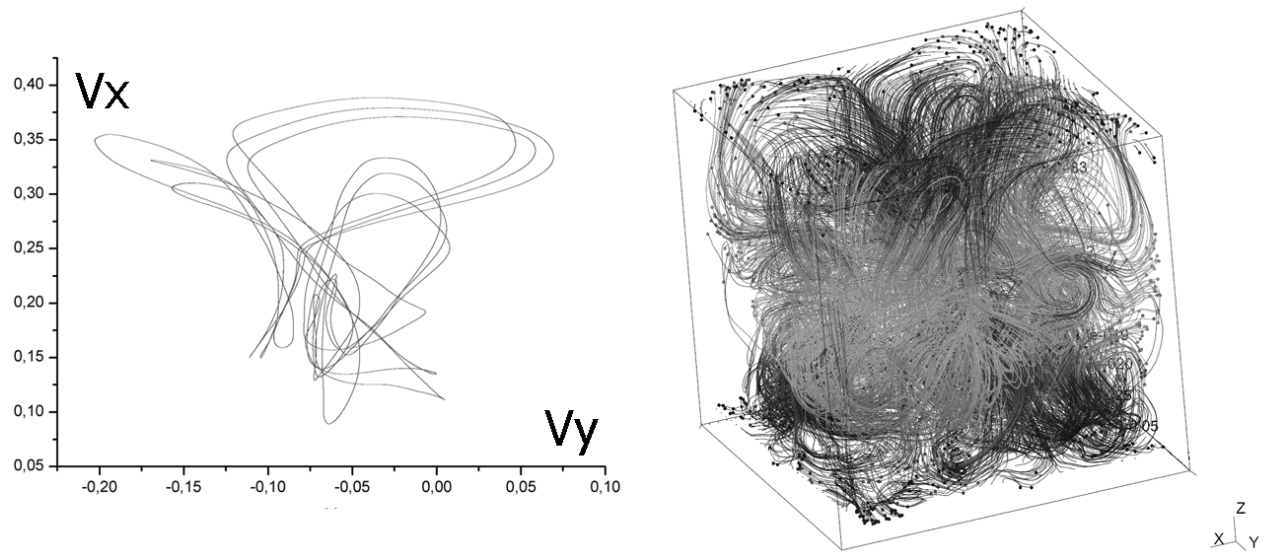

Figure 21. Triple period stable cycle at point, $\operatorname{Pr}=1.354839, R a=1.308 \cdot 10^{6}$; Stream lines in $\Omega$ for the same $\operatorname{Pr}$ and $\operatorname{Ra}$.

3. FSM scenario with subharmonic cascade of bifurcations of stable limit cycles.

\section{Conclusion}

The following chapter covers five year work that has been conducted in the Chaotic Dynamics Laboratory in the Institute for Systems Analysis of Russian Academy of Sci., lead by professor N.A. Magnitskii. Our attempt in using standard open source or commercial software for this kind of analysis failed so we had to consider a specially constructed accurate and trustworthy numerical solution code for Navier-Stokes equations, partly described here. The results of numerical solution for initial-boundary value problems considered confirmed that laminar-turbulent transition undergoing the bifurcation process and has different scenarios. It is interesting to point out that similar scenarios with classical Feigenbaum scenario and Sharkovskiy windows of periodicity where recently found in [66] for initial-boundary value problems in continuous mechanical systems such as flexible plates and shallow shells. However, the universal FSM scenario [7] is found in all problems considered, despite the difference between problems. Recently we found that Boltzmann equations in hydrodynamic limit with BGK collision integral [63-65] also exhibit FSM scenario for laminar-turbulent transition process. It is likely that all hydrodynamic type chaotic solutions for PDEs are undergoing the FSM scenario in various modifications. The work continues and now we are considering compressible fluid dynamics (transonic and supersonic turbulence) and magnetohydrodynamics as well as other initial-boundary value problems for (1).

\section{Author details}

Nikolai Magnitskii and Nikolay Evstigneev

Laboratory 11-3, Chaotic Dynamical Systems, Institute for Systems Analysis of RAS, Russian Federation 


\section{References}

[1] Carlson A., Jaffe A. and Wiles A. (Editors). (2006) The Millennium Prize Problems. Cambridge: Clay Mathematics Institute.

[2] Sadovnichii V. and Simo S.(Editors). (2002) Modern Problems of Chaos and Nonlinearity. - Moscow-Igevsk: Institute for Computational Studies.

[3] Magnitskii N.A. (2008) Universal theory of dynamical chaos in nonlinear dissipative systems of differential equations. // Commun. Nonlinear Sci. Numer. Simul., 13, pp. 416-33.

[4] Magnitskii N.A. (2007) Universal theory of dynamical and spatio-temporal chaos in complex systems. / / Dynamics of Complex Systems 1, 1, pp. 18-39. (in Russian)

[5] Magnitskii N.A. (2008) New approach to analysis of Hamiltonian and conservative systems. / /Differential Equations, 44, 12, pp. 1618-27.

[6] Magnitskii N.A. (2010) On topological structure of singular attractors of nonlinear systems of differential equations. / Differential Equations, 46, 11, pp.1551-1560.

[7] Magnitskii N.A., Sidorov S.V. (2006) New Methods for Chaotic Dynamics. - Singapure: World Scientific.

[8] Magnitskii N.A., Sidorov S.V. (2005) On transition to diffusion chaos through a subharmonic cascade of bifurcations of two-dimensional tori. / / Differential Equations , 41, 11, pp. 1550-58.

[9] Evstigneev N.M., Magnitskii N.A., Sidorov S.V. (2009) On nature of laminar-turbulent flow in backward facing step problem. / Differential equations, v.45, 1, pp.69-73.

[10] Evstigneev N.M., Magnitskii N.A., Sidorov S.V. (2009) On the nature of turbulence in Rayleigh-Benard convection./ /Differential Equations V.45, N.6, pp.909-912.

[11] Evstigneev N.M, Magnitskii N.A. and Sidorov S.V. (2010) Nonlinear dynamics of laminarturbulent transition in three dimensional Rayleigh-Benard convection.// Commun. Nonlinear Sci. Numer. Simul., 15, p. 2851-2859.

[12] Evstigneev N.M., Magnitskii N.A. (2010) On possible scenarios of the transition to turbulence in Rayleigh-Benard convection.// Doklady Akademii Nauk, Vol. 433, No. 3, pp. 318-322.

[13] Filatov A.N., Ipatova V.M. (1996) On globally stable difference schemes for the barotropic vorticity equation on a sphere // Russian J. Numer. Anal. Math. Modelling. - V. 11, N 1. - P. 1-26.

[14] Ipatova V.M. (1997) Attractors of approximations to non-autonomous evolution equations./ /Mat. Sb., Volume 188, Number 6, Pages 47-56.

[15] Foias C., Temam R.(1987) The connection between the Navier-Stokes equations, dynamical systems, and turbulence theory, in Directions in Partial Differential Equations, Academic Press, NY, 55-73

[16] Temam R. (1991) Approximation of Attractors, Large Eddy Simulations and Multiscale Methods.// Proc. R. Soc. Lond.v.434, pp23-39.

[17] Ladyzhenskaya O.A. (1985) On the finiteness of the dimention of bounded invarian sets for the Navier-Stokes equations and other related dissipative systems. / / J.Soviet Math. 28, pp714-725.

[18] Chepyzhov V., Vishik M. (2002) Attractors for Equations of Mathematical Physics.// Amer. Math. Soc. Colloq. Publ., Vol. 49, Amer. Math. Soc., Providence, RI. 
[19] Vishik M.I., Titi E.S., Chepyzhov V.V. (2007) On convergence of trajectory attractors of the 3D Navier-Stokes- $\alpha$ model as $\alpha$ approaches 0./ / SB MATH, 198 (12), pp 1703-1736.

[20] Temam R. (1983) Navier-Stokes Equations and Nonlinear Functional Analysis, SIAM.

[21] Buckingham E. (1914) On physically similar systems; illustrations of the use of dimensional equations. // Physical Review 4 pp.345-376.

[22] Evstigneev N.M. (2010) About one averaging method of the equations of a compressible and incompressible fluid. / Proc. GROU, PhM series, N2, pp.47-52. (in Russian)

[23] Evstigneev N.M., Magnitskii N.A., Sidorov S.V. (2008) New method for turbulent analysis in incompressible viscous fluid flow. // Proc. ISA RAS,v. 33, ed. 12. pp.49-65. (in Russian)

[24] Evstigneev N.M. (2008) Integration of 3D incompressible free surface Navier-Stokes equations on unstructured tetrahedral grid using distributed computation on TCP/IP networks. // Proc. Of the VII International conf. "Advances in Fluid Mechanics", pp. 194-208.

[25] Belotserkovskii O.M., Oparin A.M., Chechetkin V.M. (2005) Turbulence: New Approaches. - CISP, Science.

[26] Martin M.P. , Taylor E.M. , Wu M. , Weirs V.G. (2006) A bandwidth-optimized WENO scheme for the effective direct numerical simulation of compressible turbulence.// Journal of Computational Physics 220 (2006) 270-289.

[27] Evstigneev N.M. (2010) Numerical solution for Navier-Stokes equations on unstructured grid using semi-Lagrangian method.// Scientific bulletin of SpBSPolitechUniv., 1 (93). pp. 163-170. (in Russian)

[28] Cockburn B. and Shu C.-W. (1998) The Runge-Kutta discontinuous Galerkin method for conservation laws V: Multidimensional systems./ / J. Comput. Phys., 141, pp. 199-224.

[29] Shu C.-W. (1987) TVB uniformly high-order schemes for conservation laws.// Math. Comp., 49, pp. 105-121.

[30] Shu C.-W. and Osher S. (1988) Efficient implementation of essentially non-oscillatory shock-capturing schemes.// J. Comput. Phys., v77, pp.439-471.

[31] CUDA C Programming Guide (2011)

http://developer.nvidia.com/category/zone/cuda-zone

[32] Rani H.P., Tony W.H. Sheu (2006) Nonlinear dynamics in a backward-facing step flow./ / Physics of Fluids, Vol. 18, pp. 084101-14.

[33] Chiang T. P. and Tony W. H. Sheu. (2002) A numerical revisit of backward-facing step flow problem. / / PHYSICS OF FLUIDS, VOLUME 11, NUMBER 4, 862-874.

[34] Rani H. P., Tony W. H. Sheu and Tsai E.S.F. (2007) Eddy structures in a transitional backward-facing step flow./ / J. Fluid Mech. , vol. 588, pp. 43-58.

[35] Moin, Lee, T., Mateescu, D., (1997) Direct numerical simulation of turbulent flow over a backward-facing step.//J. of Fluids and Structures, vol. 330, pp. 349-374.

[42] Kim J., Moin P. (1985) Application of a fractional-step method to incompressible Navier-Stokes equations. / / Comp Phys, 59:308-23.

[37] Moin P., Mahesh K. (1998) Direct Numerical Simulation: A Tool in Turbulence Research./ / Annu. Rev. Fluid Mech. 30:539-78.

[38] Driver, D.M., and Jovic, S. (1998) Backward facing step - experiment. Agard advisory report, agard-ar-345, a selection of test cases for the validation of large-eddy simulations of turbulent flows.// Report CMP31. 195-196. 
[39] Adnan Meri and Wengle Hans (2004) DNS and LES of Turbulent Backward-Facing Step Flow Using 2ND-and 4TH-Order Discretization. //Fluid Mechanics and Its Applications, Volume 65, 2, 99-114.

[40] Dwight Barkley, Gabriela M., Gomes M., Henderson Ronald D. (2002) Three-dimensional instability in flow over a backward-facing step.// J. Fluid Mech., vol. 473, pp. 167-190.

[41] Varapaev, V. N. and Yagodkin, V. I. (1969) Flow stability in a channel with porous walls.// Izv. Akad. Nauk SSSR, Mekh. Zhid. i Gaza 4, 91-95.

[42] Kim J., Moin P. (1985) Application of a fractional-step method to incompressible Navier-Stokes equations. / /J Comp Phys, 59:308-23.

[43] Mouza A.A., Pantzali M.N. ,Paras S.V., Tihon J. (2005) EXPERIMENTAL and NUMERICAL STUDY OF BACKWARD-FACING STEP FLOW./ / Proc. Of 5th National Chemical Engineering Conference, Thessaloniki, Greece.

[44] Perez Guerrero J. S. and Cotta R. M. (1996) Benchmark integral transform results for flow over a backward-facing step.// Comput. Fluids 25, 527.

[45] Aider J.L. and Danet A. (2006) Large-eddy simulation study of upstream boundary conditions influence upon a backward-facing step flow.// Comptes Rendus M'ecanique, vol.334(7), 447 - 453.

[46] Jovic S., Driver D.M. (1994) Backward-facing step measurements at low Reynolds number, $R e_{h}=5000 . / /$ NASA Tech. Memo 108807.

[47] Evstigneev N.M, Magnitskii N.A. and Sidorov S.V. (2009) Nonlinear dynamics in Rayleigh-Benard convection problem.// Proc. 3d international conf. "System analysis and information technology", SAIT-2009, pp. 412-415. (in Russian)

[48] Evstigneev N.M., Ryabkov O.I. (2010) On the visualization qualitative analysis in nonlinear dynamic systems // Scientific Visualization. Electronic Journal of National Research Nuclear University "MEPhI".c.4 v.2 N.4 pp. 61-71.

[49] Volkov V. F., Tarnavskii G. A. (2001) Broken symmetry and hysteresis in steady-state and quasi-steady solutions to Euler and Navier-Stokes equations.// Zh. Vychisl. Mat. Mat. Fiz., 41:11, 1742-1750.

[50] TSAGI Web Seminar (2011)

http: //www.tsagi.ru/cgi-bin/jet/viewnews.cgi?id= 20110506121862279081

[51] Getling A.V. (1998) Rayleigh-Benard Convection. Structures and Dynamics. - Advanced Series In Nonlinear Dynamics, vol.11, World Scientific.

[52] Van Daele M., Vanden Berghe G. and De Meyer H. (1992) Five-diagonal finite difference methods based on mixed-type interpolation for a certain fourth-order two-point boundary-value problem./ / Computers and Mathematics with Applications. V24, I10, pp55-76.

[53] Diele F. (1998) The use of the factorization of five-diagonal matrices by tridiagonal Toeplitz matrices. / / Applied Mathematics Letters Volume 11, Issue 3, Pages 61-69.

[54] Evstigneev N.M. (2009) Numerical integration of Poisson's equation using a graphics processing unit with CUDA-technology.// J. Numerical Methods and Programming, V.10, pp.268-274. 
[55] Siegfried Grossmann, Detlef Lohse. (2002) Prandtl and Rayleigh number dependence of the Reynolds number in turbulent thermal convection. / / PHYSICAL REVIEW E 66, pp16305-16305-6.

[56] Siegfried Grossmann, Detlef Lohse. (2000) //J. Fluid Mech. 407, pp27-35.

[57] Castaing, B., Gunaratne G. , Heslot F., Kadanoff L., Libchaber A., et al., (1989) Scaling of hard thermal turbulence in Rayleigh- Benard convection. / / J. Fluid Mech. 204, 1-30.

[58] Niemela, J.J., Skrbek L. , Sreenivasan K.R., and Donnelly R.J.. (2000) Turbulent convection at very high Rayleigh numbers.// Nature, 398, 307-310.

[59] Evstigneev N.M. (2010) On the Lattice Boltzmann Method stabilization for turbulent flow regimes with extremely high Reynolds numbers.// Proc. GROU, PhM series, N2, pp. 53-62. (in Russian)

[60] Katarzyna Boronska, Laurette S. Tuckerman. (2010) Extreme multiplicity in cylindrical Rayleigh-Benard convection. I. Time dependence and oscillations.//PHYSICAL REVIEW E 81, 036320 pp 1-13.

[61] Watanabe T. (2004) Numerical Evidence for Coexistence of Roll and Square Patterns in Rayleigh-Benard Convetion.// Phys. Lett. A, Vol.321, pp.185-189.

[62] Frik P. G. (2003) Turbulence: Approaches and Models. - Moscow, IKI, (in Russian).

[63] Evstigneev N. M.; Magnitskii, N. A. (2010) Nonlinear Dynamics of Laminar-Turbulent Transition in Back Facing Step Problem for Bolzmann Equations in Hydrodynamic Limit.// Int. Conf. ICNAAM 2010. AIP Conference Proceedings, Volume 1281, pp. 896-900.

[64] Evstigneev N. M., Magnitskii, N. A. (2010) Nonlinear Dynamics in the Initial-Boundary Value Problem on the Fluid Flow from a Ledge for the Hydrodynamic Approximation to the Boltzmann Equations./ / Differential Equations, V.46, N.12, pp.1794-1798.

[65] Evstigneev N.M. (2010) Lattice Boltzman method with entropy stabilization on GPU. / / Proc. ISA RAS,v. 53, ed. 14. pp.49-65. (in Russian)

[66] Awrejcewicz J., Krysko V.A., Papkova I.V., Krysko A.V. (to appear 2012) Routes to chaos in continuous mechanical systems. Part 1,2,3. / / Chaos Solitons and Fractals. Nonlinear Science, Non-equilibrium and Complex Phenomena. 